\title{
Implications for tetraspanin-enriched microdomain assembly based on structures of CD9 with EWI-F
}

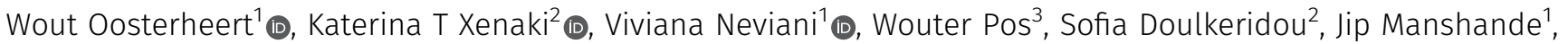

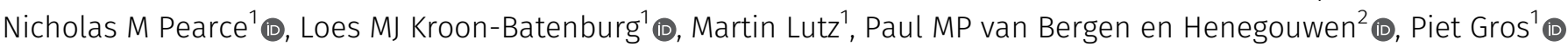

\begin{abstract}
Tetraspanins are eukaryotic membrane proteins that contribute to a variety of signaling processes by organizing partner-receptor molecules in the plasma membrane. How tetraspanins bind and cluster partner receptors into tetraspanin-enriched microdomains is unknown. Here, we present crystal structures of the large extracellular loop of CD9 bound to nanobodies 4C8 and 4E8 and, the cryo-EM structure of 4C8-bound CD9 in complex with its partner EWI-F. CD9-EWI-F displays a tetrameric arrangement with two central EWI-F molecules, dimerized through their ectodomains, and two CD9 molecules, one bound to each EWI-F transmembrane helix through CD9-helices h3 and h4. In the crystal structures, nanobodies $4 \mathrm{C} 8$ and $4 \mathrm{E} 8$ bind CD9 at loops C and D, which is in agreement with the $4 \mathrm{C} 8$ conformation in the CD9-EWIF complex. The complex varies from nearly twofold symmetric (with the two CD9 copies nearly anti-parallel) to ca. $50^{\circ}$ bent arrangements. This flexible arrangement of CD9-EWI-F with potential CD9 homo-dimerization at either end provides a "concatenation model" for forming short linear or circular assemblies, which may explain the occurrence of tetraspanin-enriched microdomains.
\end{abstract}

Dol 10.26508/lsa.202000883 | Received 18 August 2020 | Revised 4 September 2020 | Accepted 4 September 2020 | Published online 21 September 2020

\section{Introduction}

The spatial organization of proteins and lipids in the plasma membrane controls cellular processes such as signaling, trafficking, cell adhesion, and fusion (Zuidscherwoude et al, 2014). Members of the tetraspanin superfamily of transmembrane proteins act as molecular organizers of the plasma membrane by clustering specific partner proteins in cis to arrange their assembly required for function (Maecker et al, 1997; Hemler, 2005; Yáñez-Mó et al, 2009; Van Deventer et al, 2017). Tetraspanins form dynamic platforms termed tetraspanin-enriched microdomains (TEMs), also referred to as the tetraspanin web (Hemler, 2005; Yáñez-Mó et al, 2009;
Charrin et al, 2009a). TEMs were formerly described as large assemblies comprising multiple tetraspanin family members and partner proteins (Berditchevski et al, 1996; Rubinstein et al, 1996; Charrin et al, 2009a), but the most recent model derived from superresolution microscopy experiments states that TEMs are nanoclusters of $120 \mathrm{~nm}$ in diameter that contain fewer than 10 copies of a single tetraspanin homolog (Zuidscherwoude et al, 2015).

Among the 33 human tetraspanins, CD9 (also known as Tspan29) is an extensively studied tetraspanin that is expressed in a wide variety of tissues and is also highly abundant on the membranes of extracellular vesicles (Andreu \& Yáñez-Mó, 2014). CD9 participates in processes such as cell adhesion, motility, and differentiation (Yáñez-Mó et al, 2009; Chambrion \& le Naour, 2010; MachadoPineda et al, 2018). In addition, it regulates cell-cell fusion events in myoblasts (Tachibana \& Hemler, 1999; Charrin et al, 2013), osteoclasts (Ishii et al, 2006), macrophages (Takeda et al, 2003), and sperm-egg cells (Boucheix, 2000; Kaji et al, 2000). Besides its physiological functions, CD9 is widely associated with diseases: both the up-regulation and down-regulation of CD9 are linked with poor prognosis in several types of cancer, such as melanoma, leukemia, and gastric, lung, breast, colon, and prostate malignancies (Brosseau et al, 2018). Moreover, CD9 modulates HIV-1-induced membrane fusion (Gordón-Alonso et al, 2006) and promotes MERS coronavirus entry by clustering host-cell receptors and proteases (Earnest et al, 2017; Florin \& Lang, 2018).

At the molecular level, tetraspanins adopt a common architecture with four transmembrane helices (h1-h4), intracellular $\mathrm{N}$ and C-termini, a small extracellular loop between membrane helices $h 1$ and $h 2$ (EC1) and a large extracellular loop between membrane helices h3 and h4 (EC2). Initial structural studies on the soluble EC2 domain of CD81 disclosed an EC2-fold with five helical regions (termed $A-E$ ), with $A, B$, and $E$ forming a "stalk" domain and $C$ and D forming a "head" domain (Kitadokoro et al, 2001). Crystal structures of full-length tetraspanin CD81 (Zimmerman et al, 2016) and CD9 with a truncated EC2 (Umeda et al, 2020) revealed an inverted-cone shape arrangement of the transmembrane domain (TMD), with the EC2 closing off the TMD-cone like a lid, although

\footnotetext{
${ }^{1}$ Department of Chemistry, Crystal and Structural Chemistry, Bijvoet Centre for Biomolecular Research, Faculty of Science, Utrecht University, Utrecht, The Netherlands ${ }^{2}$ Department of Biology, Cell Biology, Neurobiology and Biophysics, Faculty of Science, Utrecht University, Utrecht, The Netherlands ${ }^{3}$ uniQure Biopharma, Amsterdam, The Netherlands
}

Correspondence: p.gros@uu.nl 
molecular dynamics simulations suggested that the EC2 lid can also adopt open conformations (Zimmerman et al, 2016; Umeda et al, 2020). This was recently experimentally confirmed by the crystal structure of CD53, which showed an open conformation of the EC2 (Yang et al, 2020). Previous studies have established that both the EC2 and TMD regions of tetraspanins participate in homo- and hetero-oligomeric interactions, indicating that tetraspanins use separate domain regions to bind to different partners proteins (Van Deventer et al, 2017). CD9 interacts with numerous single-span transmembrane proteins, including integrins (Rubinstein et al, 1994; Nakamura et al, 1995; Reyes et al, 2015, 2018), immunoglobin superfamily (IgSF) proteins (Charrin et al, 2001; Stipp et al, 2001a, 2001b), heparin-binding EGF-like growth factor (Iwamoto et al, 1994), and metalloprotease ADAM17 (Gutiérrez-López et al, 2011; Machado-Pineda et al, 2018). IgSF protein EWI-F, also known as CD9-partner 1 or prostaglandin F2 receptor negative regulator, is a prototypical primary CD9-binding protein that also interacts with its close-homolog CD81 in stoichiometric amounts in several cell types (Charrin et al, 2001; Stipp et al, 2001b). EWI-F comprises six extracellular, heavily glycosylated Ig-like C2 domains (André et al, 2007), a single transmembrane (TM) helix, and a small C-terminal cytoplasmic tail. Functionally, EWI-F moderates CD9 and CD81-driven cellular fusion events (Charrin et al, 2009b, 2013) and connects TEMs to the cytoskeleton by binding to ezrin-radixin-moesin proteins (Sala-Valdés et al, 2006). Biochemical experiments have revealed that the interaction between EWI-F and CD9 or CD81 is mediated by the TMDs, with critical roles for the transmembrane helix of EWI-F and helix h4 of CD9 or CD81 (André et al, 2009; Charrin et al, 2009b). In addition, we recently found that palmitoylated membrane-proximal cysteines of CD9 are crucial for maintaining the interaction with EWI-F (Neviani et al, 2020).

Although tetraspanins have a central role in human physiology, the mechanisms through which they interact with partner proteins and the molecular principles that govern the formation and signaling of TEMs remain incompletely understood. Here, we study the structural basis for interactions in the tetraspanin web using the CD9-EWI-F complex as a model system. We first present crystal structures of the isolated EC2 domain of CD9 in the absence and presence of anti-CD9 nanobodies $4 \mathrm{C} 8$ and 4E8, revealing the flexibility of the $\mathrm{D}$-loop region of $\mathrm{CD}$. We then use nanobody $4 \mathrm{C} 8$ in single-particle cryo-EM studies to show that CD9 and EWI-F form a hetero-tetramer that adopts a range of conformations, with a central EWI-F dimer flanked by two CD9 molecules on each side. The structural data are in good agreement with the recently reported cryo-EM map of CD9 bound to EWI-F homolog EWI-2 (Umeda et al, 2020), indicating that CD9 binds to EWI proteins through a common binding mode. Overall, the various conformations of the CD9-EWI-F complex observed in the cryo-EM data, combined with previous biochemical interaction studies, support a "concatenation model" for the assembly of TEMs in the plasma membrane.

\section{Results}

\section{Crystal structures of $\mathrm{CD} 9_{\mathrm{EC} 2}$ in complex with nanobodies}

Our group has previously reported the generation of several nanobodies that bind to the extracellular region of CD9 (Neviani et al, 2020). We selected nanobodies $4 \mathrm{C} 8$ and 4E8, which differ in amino-acid composition in complementarity-determining regions (CDRs) 2 and 3, for further characterization. 4C8 and 4E8 bind to purified, full-length CD9 as well as to endogenous CD9 expressed on HeLa cells with apparent binding affinities in the nanomolar range (Fig S1A-C). To determine the nanobody-binding epitopes on CD9, we solved crystal structures of the soluble EC2 of $C D 9\left(C D 9_{E C 2}\right)$ in complex with $4 \mathrm{C} 8$ at $2.7-\AA$ resolution (Fig $1 \mathrm{~A}$ ) and $4 \mathrm{E} 8$ at $1.4-\AA$ resolution (Fig 1B), as well as the structure of $4 \mathrm{C} 8$ alone at $1.7-\AA$ resolution (Fig S2A and Table 1). The $\mathrm{CD} 9_{\mathrm{EC} 2}-4 \mathrm{C} 8$ complex crystallized in space group $12_{1} 2_{1} 2_{1}$ with two copies in the asymmetric unit (Fig S2B), whereas $\mathrm{CD} 9_{\mathrm{EC} 2}-4 \mathrm{E} 8$ crystallized in the space group $\mathrm{P} 2_{1} 2_{1} 2$ with a single copy of the complex in the asymmetric unit (Fig 1B).

In both nanobody-bound structures, $\mathrm{CD} 9_{\mathrm{EC} 2}$ adopts an arrangement that is globally similar to previously reported EC2-structures of CD81 (Kitadokoro et al, 2001, 2002; Yang et al, 2015; Zimmerman et al, 2016; Cunha et al, 2017; Nelson et al, 2018). 4C8 and 4E8 bind nearly identical epitopes that span the $C$ and D-loops of CD9 ${ }_{\text {EC2 }}$ (Fig $1 A$ and $B)$. The interfaces are established through nanobody residues located in CDRs 2 and 3, whereas the CDR1 region of both nanobodies makes no contacts with $C D 9_{E C 2}$. The surface areas buried in the $\mathrm{CD}_{\mathrm{EC} 2}-4 \mathrm{C} 8$ and $4 \mathrm{E} 8$ complexes are ca. 1,280 and 1,230- $\AA^{2}$, respectively. $C D 9$ residues involved in the interfaces with the nanobodies are V159, E160, Q161, and S164 from the C loop; and K169, L173, T175, F176, and V178 from the D-loop (Fig $1 C$ and D). Both nanobodies feature an arginine residue (R101) in CDR3 that interacts with E160 through a salt bridge and two tryptophans (W53 of CDR2 and W102 of CDR3 in both 4C8 and 4E8) that form a hydrophobic core in the interface with $\mathrm{CD}_{\mathrm{EC} 2}$ (Fig $1 \mathrm{C}$ and D). In $\mathrm{CD} 9_{\mathrm{EC} 2}-4 \mathrm{E} 8$, the D-loop adopts a partially helical conformation and central residue F176 is sandwiched by 4 E8 residues W59 of CDR2 and W102 and R105 of CDR3 (Fig 1D). In the 4C8-bound $C D 9_{\mathrm{EC} 2}$ structure, the tip of the D-loop points more outward and the $C \alpha$ atom of F176 is shifted by $3 \AA$, such that F176 resides not at the center of the interface but at the periphery, where it forms a van der Waals interaction with 4C8-residue G105 (Fig 1C).

A superimposition of the nanobody-bound $\mathrm{CD} 9_{\mathrm{EC} 2}$ structures onto the crystal structure of full-length CD81 (pdb 5TCX) indicates that 4C8 and 4 E8 orient away from the membrane plane in the EC2 conformation adopted by CD81 (Fig 1E), which is consistent with both nanobodies binding to cell-membrane embedded CD9 (Fig S1A).

\section{Conformational flexibility of the $\mathrm{CD}_{\mathrm{EC} 2} \mathrm{D}$-loop}

The EC2 D-loop displays a high amino-acid sequence variability among tetraspanins and has been proposed to mediate homo- and hetero-oligomeric interactions (Homsi et al, 2014; Schmidt et al, 2016; Homsi \& Lang, 2017; Van Deventer et al, 2017). Previously reported structures of $C D 81$ revealed the conformational plasticity of its EC2 D-loop, with both fully helical and partially unfolded arrangements (Kitadokoro et al, 2001, 2002; Yang et al, 2015; Zimmerman et al, 2016; Cunha et al, 2017; Nelson et al, 2018). Our nanobody-bound $\mathrm{CD}_{\mathrm{EC} 2}$ structures also display a minor conformational difference in D-loop with respect to each other (Fig 1C and D). To further investigate the conformational changes adopted by the D-loop of CD9 upon nanobody binding, we determined the crystal structure of $C D 9_{\mathrm{EC} 2}$ in the absence of nanobodies at $2.0-\AA$ resolution (Table 1). The diffraction data indicated non-merohedral 
A

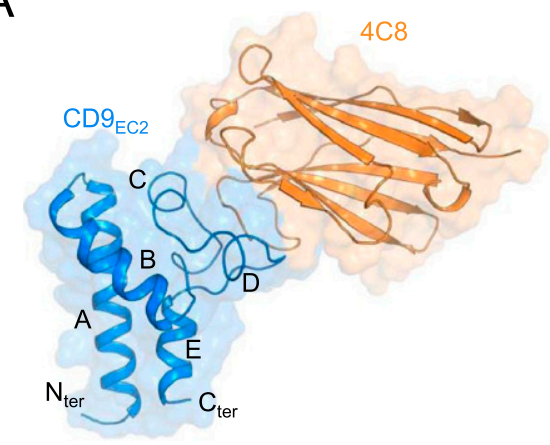

B

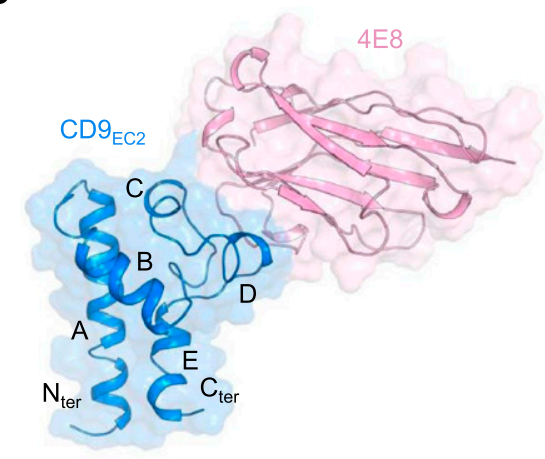

C

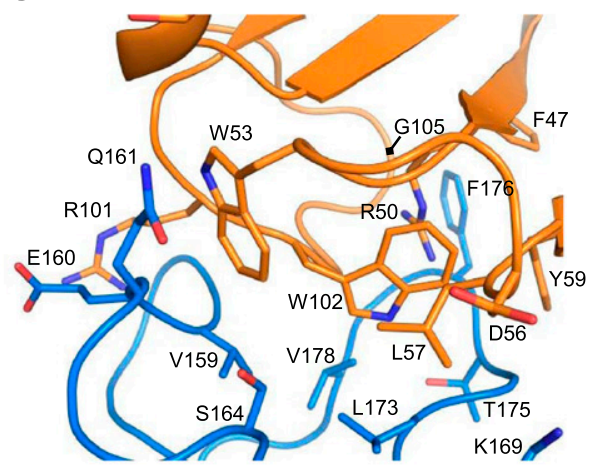

D

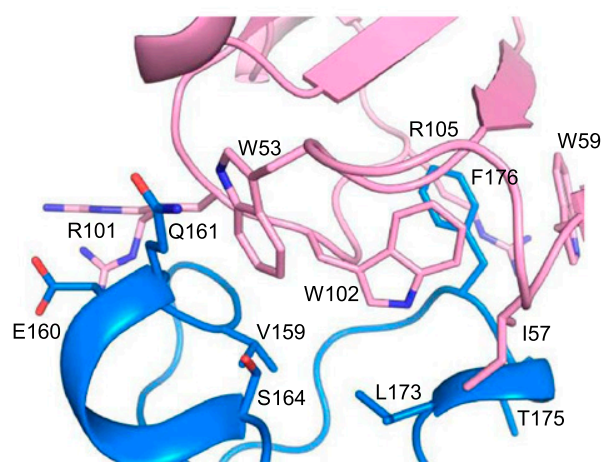

$E$

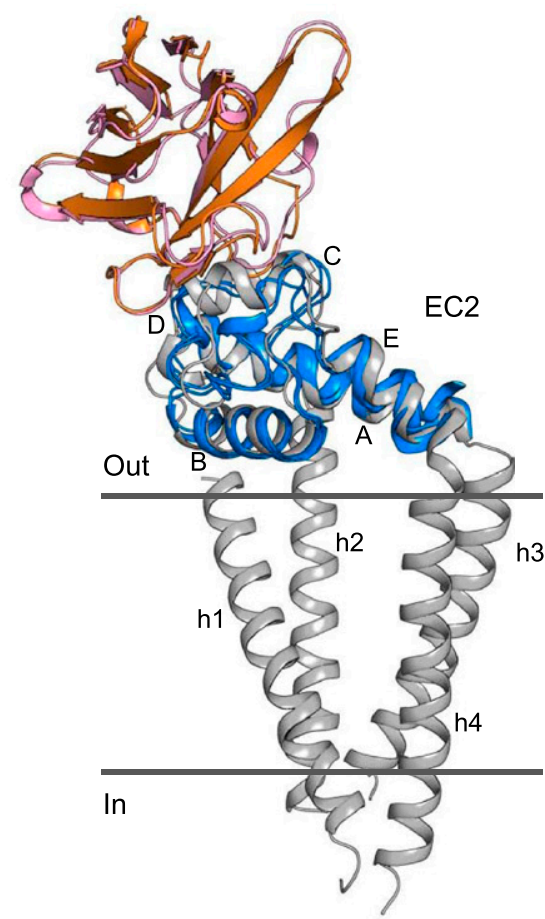

Figure 1. Nanobody-bound $\mathrm{CD} 9_{\mathrm{EC} 2}$ structures.

(A, B) Crystal structure of $\mathrm{CD} 9_{\mathrm{EC} 2}$ (blue) bound to nanobody $4 \mathrm{C} 8$ (orange, panel A) and nanobody $4 \mathrm{E} 8$ (pink, panel B). The regions of the EC2 are annotated. (C, D) Interaction interface between $\mathrm{CD} 9_{\mathrm{EC} 2}$ (blue) and nanobody $4 \mathrm{C} 8$ (orange, panel C) and nanobody $4 \mathrm{E} 8$ (pink, panel D). Residues contributing to the interface are shown as sticks. (E) Overlay of the nanobody-bound EC2 structures with the structure of full-length CD81 (gray). The CD81 structure is shown parallel to the membrane as a side view.

twinning of the crystal with a twofold rotation around the $a^{*}+b^{*}$ diagonal as the twinning operation (Fig S3). Moreover, we observed diffuse streaks in the $a^{*}+b^{*}$ direction (Fig S3A). Fig S3B shows the two twin domains in the crystal with the twinning interface in the middle. For structure refinement, the data were de-twinned based on the calculated structure factors (see the Materials and Methods section) (Supplemental Data 1).

$\mathrm{CD}_{\mathrm{EC} 2}$ crystallized in space group P1 with four molecules in the asymmetric unit, arranged as a dimer of domain-swapped dimers (Fig 2A). In this domain swap, the $\mathrm{N}$-terminal $\mathrm{A}$ helix (residues 114-138) is exchanged with the A helix of its dimeric partner, resulting in an extensive interface that buries ca. $3,400-\AA^{2}$ of surface area. The two connected protein cores each strongly resemble the monomeric form of $\mathrm{CD} 9_{\mathrm{EC} 2}$, which is commonly observed for structures of domain-swapped dimers (Bennett et al, 1994; Liu \& Eisenberg, 2002). The domain-swapped dimers are packed in the crystal lattice through interactions between the D-loop regions, which are extended and fully unfolded (Fig 2A). A comparison between all $C D 9_{\mathrm{EC} 2}$ molecules in the asymmetric unit indicated that the D-loop adopts two distinct conformations, both different from the nanobody-bound $\mathrm{CD} 9_{\mathrm{EC} 2}$ structures (Fig 2B). This observation suggests that the binding of nanobodies $4 \mathrm{C} 8$ and $4 \mathrm{E} 8$ to $\mathrm{CD} 9_{\mathrm{EC} 2}$ changes the D-loop conformation. This may, at least in part, explain the difference in signal intensity between $4 \mathrm{C} 8$ and $4 \mathrm{E} 8$ binding to purified CD9 and to CD9 on cells (Fig S1A and B), assuming that the particular conformational change in the D-loop of CD9 is less efficient in the case of $4 \mathrm{E} 8$.

An overlay of the $\mathrm{C}$ - and $\mathrm{D}$-loop regions of $\mathrm{CD} 9_{\mathrm{EC} 2}$ structures in the absence and presence of nanobodies and full-length CD81 (pdb 5TCX) revealed a diverse set of D-loop conformations: the D-loop of CD9 ${ }_{\mathrm{EC} 2}$ is extended $\sim 11 \AA$ compared with the compact helical arrangement observed in the structure of full-length CD81 (Fig 2B). In the twinned $\mathrm{CD} 9_{\mathrm{EC} 2}$ structure, one of the D-loops has large B-factors (Fig S3C), indicating its flexibility, and it is involved in the twinning interface, apparently accommodating the deviation of true twofold symmetry of the two independent molecules. In addition, the C-loops of the $\mathrm{CD} 9_{\mathrm{EC} 2}$ structures adopt a more loop-like conformation compared with the helical C-loops present in CD81 structures (Fig 2B).

\section{Purified CD9-EWI-F complex is flexible}

We next focused on obtaining a structural model of full-length CD9 in complex with its primary partner EWI-F. To this end, we coexpressed Strep-GFP-tagged CD9 together with EWI-F in HEK293 cells and isolated the complex in N-dodecyl-B-D-maltoside (DDM) detergent using Strep-affinity chromatography and size-exclusion chromatography (SEC) purification steps (Fig 3A and B). The complex did not dissociate during subsequent analytical fluorescence assisted SEC experiments (Fig 3C), indicating that the interaction between CD9 and EWI-F is preserved outside the native cell-membrane 
Table 1. Crystallographic and structure refinement statistics.

\begin{tabular}{|c|c|c|c|c|}
\hline & CD9 $9_{\mathrm{EC} 2}$ PDB 6RLR & $\mathrm{CD} 9_{\mathrm{EC} 2}-4 \mathrm{C} 8$ PDB $6 Z 20$ & $\mathrm{CD}_{\mathrm{EC2}}-4 \mathrm{E} 8$ PDB $6 \mathrm{Z1V}$ & 4C8 PDB $6 Z 1 Z$ \\
\hline Data collection & DLS I-04 & DLS I-03 & DLS I-04-1 & ESRF ID23-2 \\
\hline Wavelength $(\AA)$ & 0.9795 & 0.9763 & 0.9159 & 0.8731 \\
\hline Resolution range $(\AA)^{\mathrm{a}}$ & $29.02-2.0(2.07-2.0)$ & $88.49-2.68(2.83-2.68)$ & $44.72-1.33(1.44-1.33)$ & $24.64-1.70(1.76-1.70)$ \\
\hline Space group & $P 1$ & I 212121 & P 21212 & P 1211 \\
\hline Cell dimensions a, b, c $(\stackrel{\AA}{A})$ & $39.99,39.40,63.64$ & $75.65,121.42,129.25$ & $61.52,89.45,35.68$ & $48.91,41.42,56.86$ \\
\hline Cell angles $\alpha, \beta, \gamma\left(^{\circ}\right)$ & $80.93,76.29,68.15$ & $90.0,90.0,90.0$ & $90.0,90.0,90.0$ & $90.0,89.7,90.0$ \\
\hline Total reflections ${ }^{a}$ & $76,175(6,985)$ & $95,756(5,440)$ & $205,399(9,736)$ & $78,129(7,815)$ \\
\hline Unique reflections $^{a}$ & $22,863(2,180)$ & $14,256(750)$ & $32,044(1,603)$ & $23,719(2,338)$ \\
\hline Completeness (spherical) $)^{\mathrm{a}}$ & $95.6(93.0)$ & $83.5(26.3)$ & $69.8(17.1)$ & $94.1(93.4)$ \\
\hline Completeness (ellipsoidal)a & $\mathrm{n} / \mathrm{a}$ & $93.9(55.8)$ & $92.0(68.9)$ & $\mathrm{n} / \mathrm{a}$ \\
\hline \multirow{3}{*}{$\begin{array}{l}\text { Diffraction limits and eigenvectors of ellipsoid fitted to } \\
\text { diffraction cutoff surface }(\AA)\end{array}$} & $\mathrm{n} / \mathrm{a}$ & $a *: 2.60$ & $a *: 1.71$ & $\mathrm{n} / \mathrm{a}$ \\
\hline & & $b^{*}: 2.72$ & $b^{*}: 1.40$ & \\
\hline & & $c^{*}: 3.04$ & $c^{*}: 1.30$ & \\
\hline Multiplicity $^{a}$ & $3.4(3.4)$ & $6.7(7.3)$ & $6.4(6.1)$ & $3.4(3.4)$ \\
\hline$|/ \sigma|^{a}$ & $4.8(0.8)$ & $9.5(1.1)$ & $17.6(1.5)$ & $4.1(0.8)$ \\
\hline$R_{\text {merge }}{ }^{a}$ & $0.10(1.15)$ & $0.13(1.78)$ & $0.04(0.98)$ & $0.129(1.057)$ \\
\hline$R_{\text {pim }}{ }^{\mathrm{a}}$ & $0.07(0.73)$ & $0.05(0.71)$ & $0.02(0.60)$ & $0.083(0.677)$ \\
\hline $\mathrm{CC}_{1 / 2}{ }^{\mathrm{a}}$ & $0.998(0.322)$ & $0.997(0.507)$ & $1.000(0.653)$ & $0.993(0.429)$ \\
\hline \multicolumn{5}{|l|}{ Refinement } \\
\hline Reflections used in refinement & 22,858 & 14,242 & 32,041 & 23,706 \\
\hline$R_{\text {work }} / R_{\text {free }}$ & $0.239 / 0.279$ & $0.206 / 0.248$ & $0.151 / 0.190$ & $0.201 / 0.231$ \\
\hline No. of non-hydrogen atoms & 2,569 & 3,123 & 1,854 & 1,967 \\
\hline Macromolecules & 2,525 & 3,104 & 1,702 & 1,827 \\
\hline Ligands & $\mathrm{n} / \mathrm{a}$ & 13 & 18 & $\mathrm{n} / \mathrm{a}$ \\
\hline Solvent & 44 & 6 & 134 & 140 \\
\hline Average $B$-factor $\left(A^{2}\right)$ & 41.7 & 68.9 & 27.0 & 25.3 \\
\hline Macromolecules & 41.8 & 69.0 & 26.5 & 24.8 \\
\hline Ligands & $\mathrm{n} / \mathrm{a}$ & 69.0 & 57.8 & $\mathrm{n} / \mathrm{a}$ \\
\hline Solvent & 36.1 & 50.6 & 38.9 & 31.3 \\
\hline \multicolumn{5}{|l|}{ R.m.s. deviations } \\
\hline Bond lengths $(\stackrel{\AA}{A})$ & 0.010 & 0.003 & 0.008 & 0.002 \\
\hline Bond angles $\left({ }^{\circ}\right)$ & 1.75 & 0.50 & 0.94 & 0.55 \\
\hline \multicolumn{5}{|l|}{ Ramachandran plot } \\
\hline Favored (\%) & 99.4 & 94.6 & 97.5 & 97.4 \\
\hline Allowed (\%) & 0.6 & 5.1 & 2.5 & 2.2 \\
\hline Disallowed (\%) & 0.0 & 0.3 & 0.0 & 0.4 \\
\hline Rotamer outliers (\%) & 4.9 & 6.4 & 1.1 & 0.0 \\
\hline Clashscore & 3.99 & 2.94 & 3.22 & 2.58 \\
\hline
\end{tabular}

*Highest resolution shell in parentheses.

$5 \%$ of reflections were used for calculation of $R_{\text {free }}$.

environment. We then collected a preliminary single-particle cryo-EM dataset of the purified complex. The resulting micrographs showed elongated, highly heterogeneous protein particles (Fig 3D). This heterogeneity is likely caused by flexibility between the six Ig-like C2 domains of
EWI-F, indicating that the imaged CD9-EWI-F complex in its full-length version is unsuitable for high-resolution structure determination. 2D classification experiments yielded low-resolution class averages with no distinguishable micelle region present. Nevertheless, a 
A

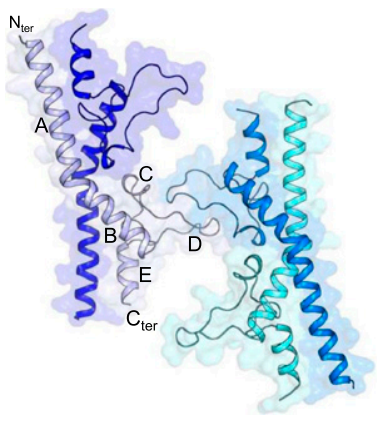

B

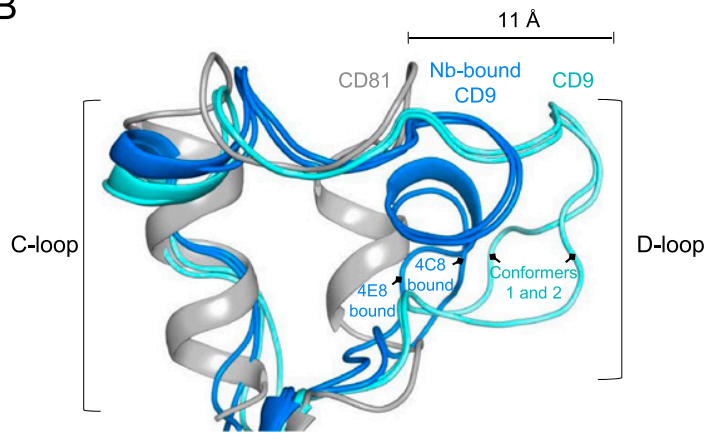

Figure 2. $\quad \mathrm{CD} 9_{\mathrm{EC} 2}$ structure and D-loop flexibility. (A) Asymmetric unit of the twinned $\mathrm{CD} 9_{\mathrm{EC} 2}$ crystal, colored by protein chain. The regions of a single EC2 chain are annotated. (B) Overlay of the C- and D-loop arrangements of $\mathrm{CD}_{\mathrm{EC} 2}$ (cyan), nanobody $(\mathrm{Nb}) 4 \mathrm{C} 8$ and $4 \mathrm{E} 8$ bound $\mathrm{CD}_{\mathrm{EC} 2}$ (blue), and full-length $\mathrm{CD} 81$ (gray, pdb 5TCX) single 2D-class average showed two rows of five domain-like densities stacked together (Fig 3E, boxed in red), presumably corresponding to five of the six Ig-like domains of EWI-F. This suggests that EWI-F forms dimeric assemblies, which is consistent with a previous cross-linking study (André et al, 2009).

\section{CD9 interacts with truncated EWI-F variants}

Because full-length EWI-F displayed severe inter-domain flexibility, we next aimed to create a structurally more homogeneous EWI-F protein sample. For this purpose, we designed and generated
A

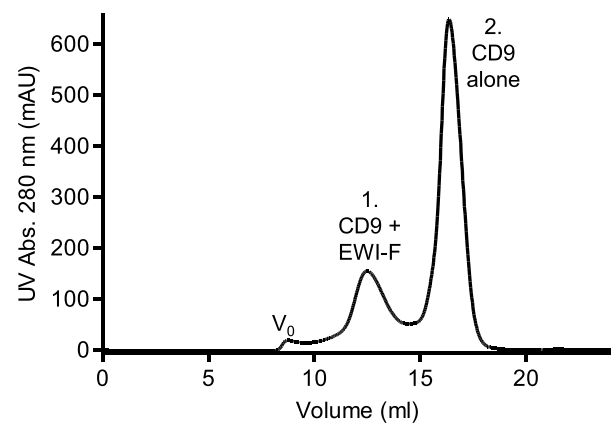

D

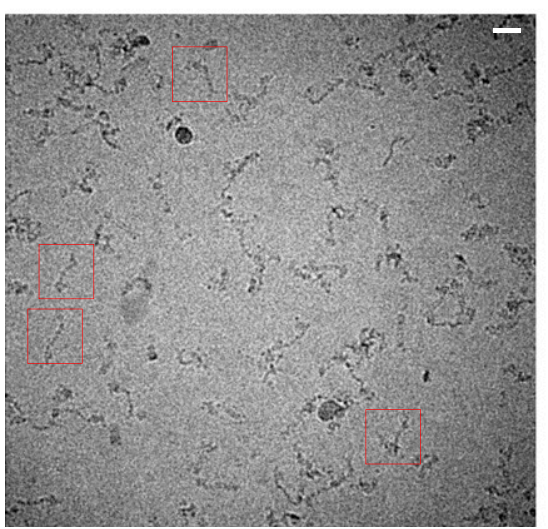

B

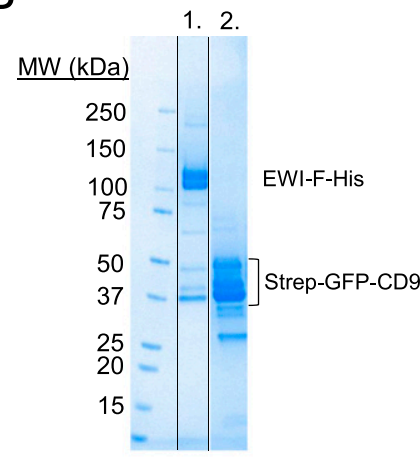

$E$

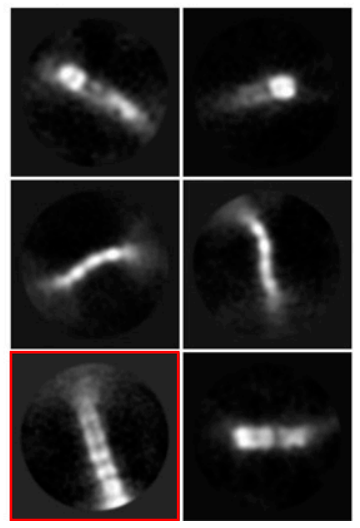

C

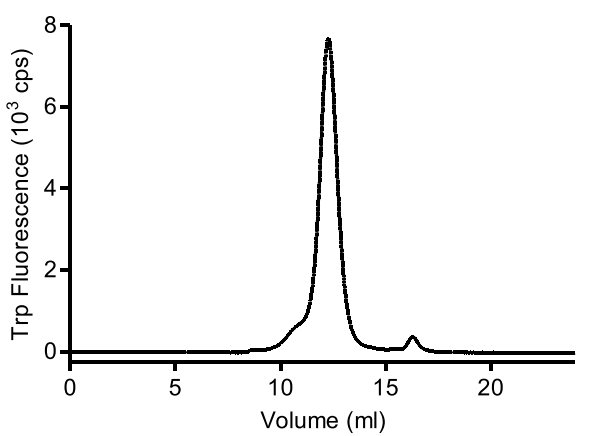

$\mathrm{F}$

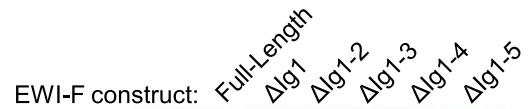

$\frac{\mathrm{MW}}{(\mathrm{kDa})} 250$

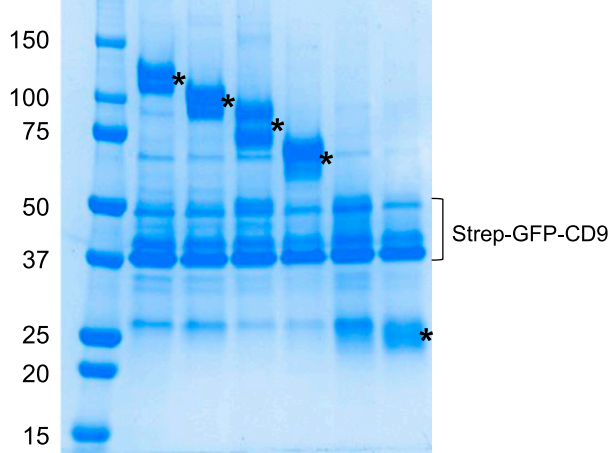

Figure 3. Biochemical and structural characterization of CD9 with full-length EWI-F and EWI-F truncations.

(A) Size-exclusion chromatography (SEC) elution profile of co-expressed CD9 and EWI-F after Strep-affinity purification. (B) SDS page gel of peaks 1 and 2 of the SEC elution from panel (A). Multiple bands are visible for EWI-F because of heterogeneous glycosylation and for CD9 because of the partial SDS-induced unfolding of the GFPtag. (C) Analytical tryptophan fluorescence-assisted SEC elution profile of the CD9-EWI-F complex used for cryo-EM. (D) Cryo-EM micrograph depicting CD9-EWI-F particles in vitreous ice. The scale bar length is $200 \AA$. Examples of individual particles are boxed in red. (E) Selected 2D-class averages generated through Relion. The box size is $409 \times 409 \AA$. The class that shows evidence for dimeric EWI-F is boxed in red. (F) SDS page gel of Strep-purified CD9 with different variants of EWI-F. Gel bands at the expected molecular weights of the EWI-F variants are marked with an asterisk $\left(^{*}\right)$. 
$\mathrm{N}$-terminally truncated EWI-F constructs with up to five Ig-like domains removed, which we termed $E W I-F_{\Delta \mid g 1}, E W I-F_{\Delta \mid g 1-2}, E W I-F_{\Delta \mid g 1-3}, E W I-F_{\Delta \mid g 1-4 \text {, }}$ and EWI-F $F_{\Delta \mid g 1-5}$. To assess if the truncated EWI-F constructs still associated with $C D 9$, we expressed the EWI-F variants together with Strep-GFP-tagged CD9 in small scale HEK293-cell cultures and, after Strep-affinity purification, monitored the amount of co-purified EWI-F using SDS-PAGE. The SDS-PAGE gel revealed the presence of protein bands at expected molecular weights for all EWI-F variants except for $E W I-F_{\Delta \mid g 1-4}$ (Fig 3F), suggesting that $E W I-F_{\Delta \mid g 1-4}$ was possibly not expressed. Nevertheless, the co-purification of EWI- $\mathrm{F}_{\triangle \mathrm{Ig} 1-5}$ with $\mathrm{CD} 9$ indicates that the first five Ig-like domains of EWI-F are not essential for maintaining the CD9-EWI-F interaction. This is in agreement with previous studies that identified the transmembrane helix of EWI-F as the main CD9 and CD81-interacting region (André et al, 2009; Charrin et al, 2009b). Based on these co-purification experiments, we chose to perform our subsequent cryo-EM experiments with construct EWI- $F_{\Delta \mid g 1-5 \text {, }}$ which harbors only a single Ig-like domain (Ig6) and thus presumably has the least conformational freedom of all tested EWI-F constructs.

\section{Cryo-EM of EWI-F $\mathrm{AIg1-5}_{-\mathrm{CD} 9-4 \mathrm{C} 8 \text { complex }}$}

We next expressed Strep-tagged, full-length CD9 (without GFP) together with $\mathrm{EWI}-\mathrm{F}_{\triangle \mathrm{Ig} 1-5}$ and purified the complex in digitonin in sufficient quantities for cryo-EM experiments. An EWI-F $\mathrm{Alg1-5}_{\mathrm{g}}$ dimer in complex with one or two CD9 molecules comprises <100 kD of protein mass, making it a challenging sample for cryo-EM. We, therefore, investigated whether nanobody $4 \mathrm{C} 8$ could be used to create a larger protein particle with more extra-membrane (or extra-micelle) features. To determine if nanobody $4 \mathrm{C} 8$ could still bind CD9 complexed to EWI-F $F_{\triangle I 91-5}$, we incubated the complex with an excess of $4 \mathrm{C} 8$ and subjected it to a SEC-purification step. The resulting $S E C$-elution profile showed a monodisperse peak for the

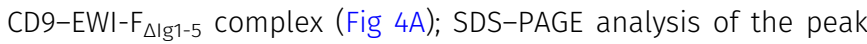
then confirmed the presence of $4 \mathrm{C} 8$ (Fig 4B), indicating that the epitope recognized by $4 \mathrm{C} 8$ is still accessible and that $4 \mathrm{C} 8$ does not disrupt the association between EWI-F $F_{\triangle \mid g 1-5}$ and CD9. The EWI- $F_{\triangle \mid g 1-5^{-}}$ CD9-4C8 complex was monodisperse in size as assessed by Trpfluorescence assisted SEC (Fig 4C), and thus suitable for analysis by single-particle cryo-EM. We collected a cryo-EM dataset and observed homogeneously sized protein particles distributed in vitreous ice (Fig 4D), which allowed more accurate particle picking than for the previous dataset with full length EWI-F (Fig 3D). 2Dclass averages of the picked particles revealed expected density features, with protein domains protruding from a disk-shaped micelle region (Fig 4E). Image processing in Relion yielded reconstructed density maps at a maximum global resolution of $\sim 8.6$
A

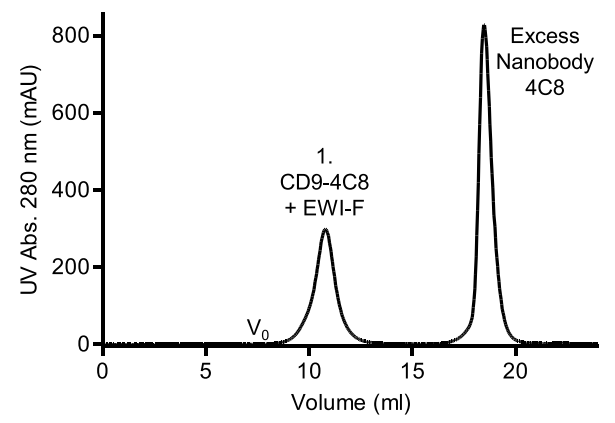

B

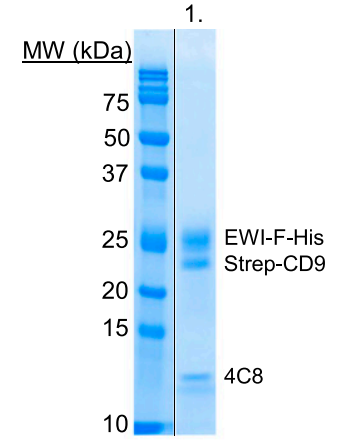

C

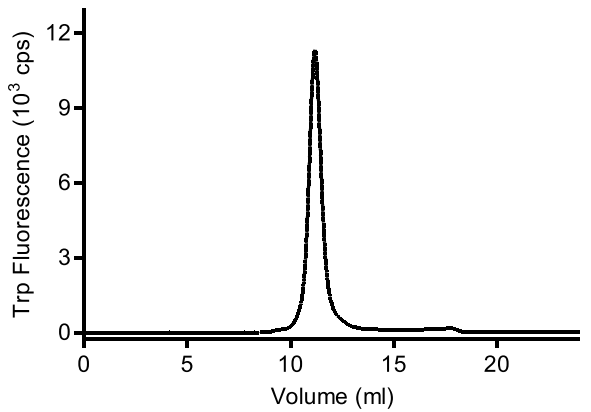

D

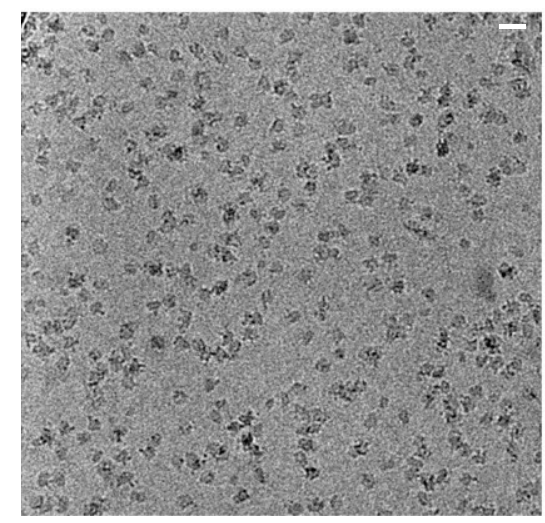

E

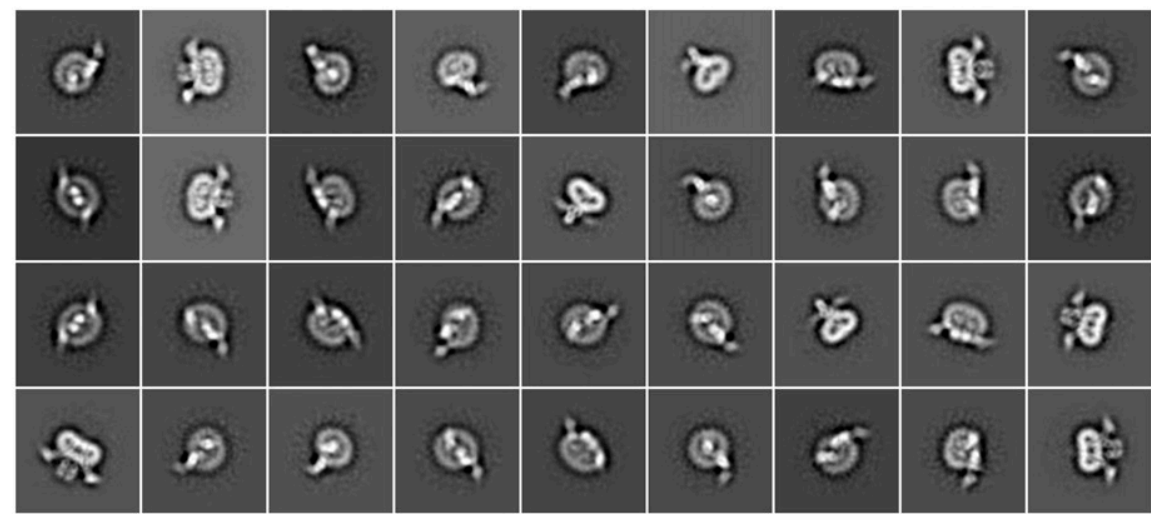

Figure 4. Cryo-EM sample preparation and imaging of EWI- $F_{\triangle I g 1-5}-C D 9-4 C 8$.

(A) Size-exclusion chromatography (SEC) elution profile of co-expressed CD9 and EWI-F $\mathrm{F}_{\Delta 1-5}$, after preincubation with a large excess of nanobody 4C8. (B) SDS page gel of peaks 1 of the SEC elution from panel (A). (C) Analytical fluorescence-assisted SEC elution profile of the EWI-F $\mathrm{F}_{\triangle \mathrm{g} 1-5}-\mathrm{CD} 9-4 \mathrm{C} 8 \mathrm{Complex}$ used for Cryo-EM. (D) Cryo-EM micrograph depicting EWI- $\mathrm{F}_{\triangle I \mathrm{~g} 1-5}-\mathrm{CD} 9-4 \mathrm{C} 8$ particles in vitreous ice. The scale bar length is $200 \AA$. (E) Selected $2 \mathrm{D}$-class averages generated through Relion. The box size is $309 \times 309 \AA$. 
$\AA$ (Figs 5A-C and S4A-D and Table 2), although protein regions of the map exhibit a higher local resolution (Fig S4B).

\section{Complex architecture and flexibility}

The $\sim 8.6-\AA$ resolution cryo-EM map revealed a hetero-tetrameric arrangement of CD9-EWI-F $F_{\triangle \mid g 1-5}$, with a central EWI-F $F_{\triangle \mid g 1-5}$ dimer flanked by 4C8-bound CD9 on each side (Fig 5A-C). Although the resolution of the reconstruction did not allow for de novo modeling, density regions corresponding to protein domains of 4C8-bound CD9 and EWI-F could be distinguished, and rigid-body fits of structures of $C D 9_{E C 2}-4 C 8$ (Fig 1A), the TMD of CD9 (pdb 6K4J) and homology models for the EWI-F Ig6 (chain b of pdb 1F3R) and TMhelix ( $p d b 5 \mathrm{EH} 4$ ) were in good agreement with the density map (Fig $5 A-C)$. Consistent with a TMD interface between CD9 and EWI- $F_{\triangle I g 1-5}$ (André et al, 2009; Charrin et al, 2009b), we observed that the EWI-F TM-helix resides in close proximity to CD9-helix h3 at the membrane center and to CD9-helix h4 at the cytoplasmic side of the membrane (Fig 5D). The two TMD regions of the complex, each comprising the four helices of CD9 and the TM helix of EWI-F, are separated and do not interact, but instead are bridged by the extracellular dimeric Ig6 domain of the two EWI-F molecules.
Overall, CD9 and EWI-F share no extensive interface through their extra-membrane domains, with no major contact areas between the EC2 domains of CD9 and EWI-F-Ig6. The 4C8-bound C and D-loops of the EC2 orient away from EWI-F. Compared with the structure of $\mathrm{CD} 81$, the $\mathrm{EC} 2$ is rotated upward by $\sim 40^{\circ}$, resulting in an open conformation of CD9 when in complex with EWI-F (Fig 5E).

Recently, a cryo-EM density map has been reported of CD9 in complex with an EWI-F homolog, EWI-2 (Umeda et al, 2020). Both the CD9-EWI-2 map and our CD9-EWI-F map were solved at moderate resolutions, limiting a detailed comparison of both reconstructions. However, both reconstructions reveal a similar hetero-tetrameric complex, with a central EWI-protein dimer and two CD9 molecules on each side (Fig S5). Thus, CD9 interacts with its major partners EWI-F and EWI-2 in a comparable protein arrangement.

During the EM image processing, it became apparent that the imaged EWI-F $F_{\triangle I g 1-5}-C D 9-4 C 8$ complex adopts a range of conformations. A typical 3D classification experiment with four major

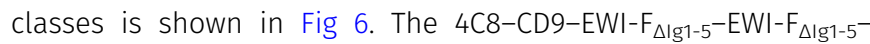
CD9-4C8 composition suggests that the complex might arrange as a C2-symmetric particle. However, we observed no twofold rotation axis in any of the four classes shown in Fig 6, nor in any further subclassifications. Class \#1 resembles the orientation of the map
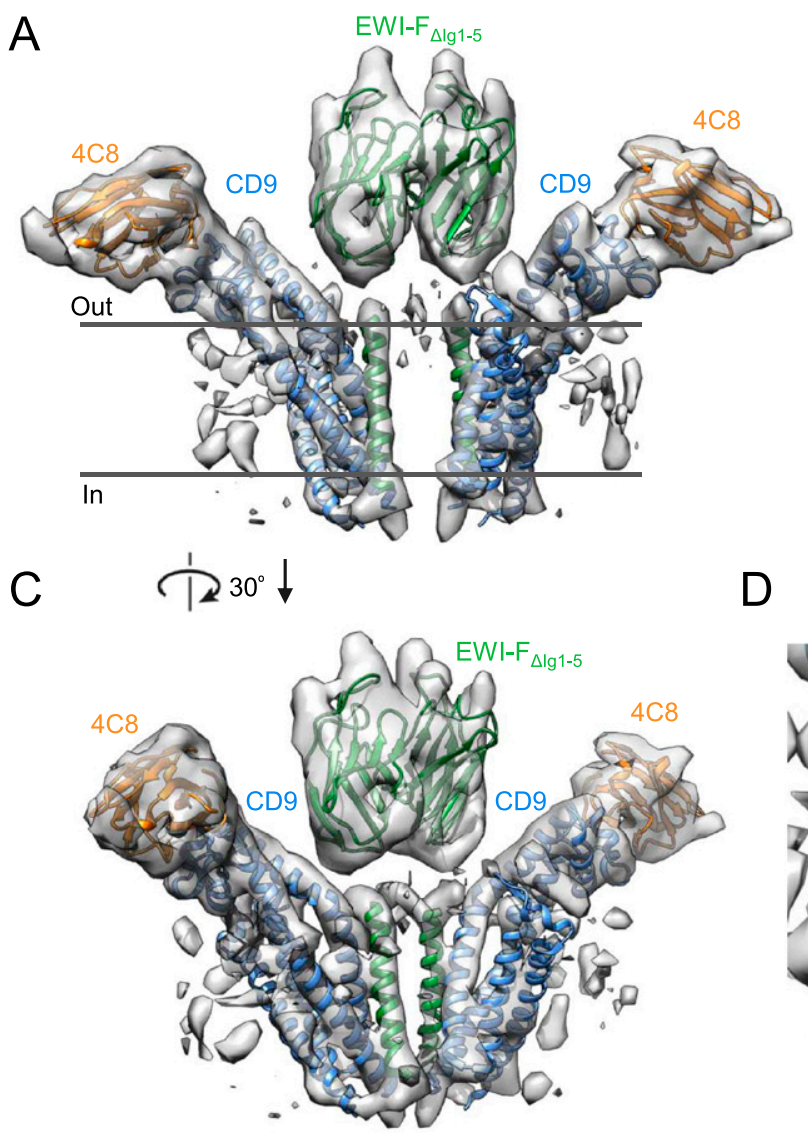

B

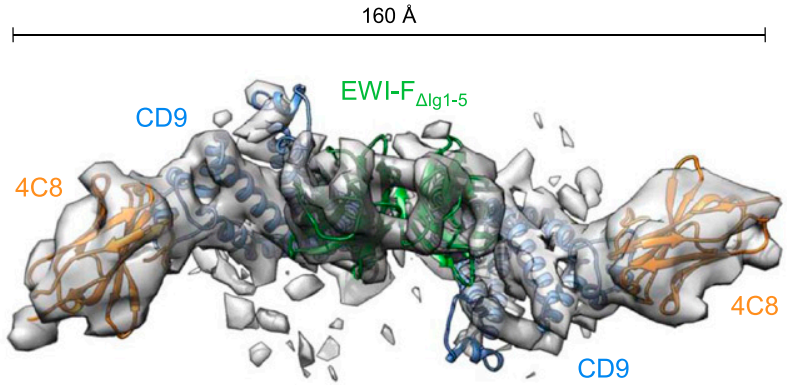

Figure 5. Cryo-EM structure of EWI- $\mathrm{F}_{\triangle \mathrm{Ig1} 1-5}-\mathrm{CD} 9-4 \mathrm{C} 8$.

(A, B, C) Sharpened, local-resolution filtered cryo-EM density map of EWI-F $F_{\triangle 191-5}-C D 9-4 C 8$ fitted with structures of CD9 EC2 $^{-4 C 8}$, the TMD of CD9 (6K4)) and homology models of EWI-F, as viewed parallel to the membrane as a side view (A), orthogonal to the membrane from the extracellular side (B), or as a side view rotated by $30^{\circ}$ (C). (D) Zoom of the major interaction region in a CD9 - EWI-F hetero-dimer. Membrane helices are annotated. (E) Overlay of the 4C8-bound CD9 EC2 $_{\text {as }}$ oriented in the Cryo-EM structure with the structure of CD81 (gray, pdb 5TCX). 
Table 2. Cryo-EM data collection and processing.

\begin{tabular}{|c|c|}
\hline Data collection and processing & EWI-F $F_{\Delta I 91-5}-C D 9-4 C 8$ EMDB-11053 \\
\hline Microscope & Talos Arctica \\
\hline Camera & Gatan K2 Summit + GIF \\
\hline Magnification & 130,000 \\
\hline Voltage (kV) & 200 \\
\hline Exposure time frame/total (s) & $0.2 / 7.2$ \\
\hline Number of frames & 36 \\
\hline Electron exposure $\left(\mathrm{e}^{-} / \AA^{2}\right)$ & 52.0 \\
\hline Defocus range $(\mu \mathrm{m})$ & -0.8 to -3.0 \\
\hline Pixel size $(\AA)$ & 1.029 \\
\hline Symmetry Imposed & $\mathrm{C} 1$ \\
\hline Micrographs (no.) & 10,724 \\
\hline Initial particle images (no.) & $1,148,718$ \\
\hline Final particles images (no.) & 354,272 \\
\hline Map Resolution $(\AA)$ & \multirow{2}{*}{8.57} \\
\hline $0.143 \mathrm{FSC}$ threshold & \\
\hline Map resolution range $(\AA)$ & $7.9-17.4$ \\
\hline
\end{tabular}

presented in Fig $5 \mathrm{~A}$ and shows a nearly twofold arrangement, with a $9^{\circ}$ deviation in anti-parallel orientation of the two 4C8-bound CD9 copies in the complex. In contrast, classes \#2-\#4 show a much larger deviation from an anti-parallel CD9 configuration, that is, a deviation of $49^{\circ}$ is observed in class \#4. A morph of 4C8-bound CD9 models fitted in the different classes reveals the putative conformational changes of CD9 in the complex (Video 1).

\section{Discussion}

Tetraspanin-mediated interactions that drive the clustering of single-pass membrane receptors into TEMs in plasma membranes have remained obscure. Structural insights into tetraspanins have so far encompassed the large extracellular EC2 loop of CD81 (Kitadokoro et al, 2001, 2002; Yang et al, 2015; Cunha et al, 2017; Nelson et al, 2018), full-length CD81 (Zimmerman et al, 2016) and, more recently, full-length CD53 (Yang et al, 2020), full-length CD9, and a low-resolution cryo-EM map of CD9 in complex with EWI-2 (Umeda et al, 2020).

The three crystal structures, presented here, reveal an overall architecture of $\mathrm{CD} 9_{\mathrm{EC} 2}$ similar to that of the homologous $\mathrm{CD} 81_{\mathrm{EC} 2}$, except for a more extended arrangement of the C-loop and, in particular, D-loop regions. In structures of CD81, these regions adopt more helical conformations, although partially unfolded arrangements are also observed in antibody-bound CD81 $1_{\mathrm{EC} 2}$ structures (Nelson et al, 2018). The D-loop conformations in CD9 $9_{E C 2}$ range from a partial helical arrangement in the nanobody-bound structures to an extended loop with no secondary structure elements in the domain-swapped $\mathrm{CD}_{\mathrm{EC} 2}$ structure without nanobody (Fig 2B). The homo-dimerizing D-loops in between two domainswapped $C D 9_{\mathrm{EC} 2}$ dimers (Fig 2A) could explain the proposed homodimerization interface in the homologous CD81, as mediated by
D-loop residues P176- F186 (Homsi et al, 2014; Schmidt et al, 2016; Homsi \& Lang, 2017) (which correspond to P168-T177 in CD9). Overall, the ability of the D-loop, which is sequence variable among tetraspanins, to adopt multiple conformations is preserved in the structures of $\mathrm{CD} 9_{\mathrm{EC} 2}$ and $\mathrm{CD} 81_{\mathrm{EC} 2}$. This observation supports the hypothesis that the EC2 head region can be tailored to facilitate interactions with specific partner proteins (Seigneuret et al, 2001). In the full-length crystal structure of CD81, molecules pack in an anti-parallel fashion through hydrophobic interaction of their TM regions, resulting in a non-physiological up-down arrangement (Zimmerman et al, 2016). Although the molecules in the crystal structure of full-length CD9 (Umeda et al, 2020) align in a more physiologically relevant manner, potential homo-dimerizing contacts between EC2 fragments were abrogated because of the deletion of D-loop residues T175-K179 (Umeda et al, 2019). When taken together, the variable D-loop conformations observed in crystal structures are consistent with the representation of the D-loop as an "interaction hub" for homo- and hetero-oligomerization in the head region of tetraspanins, as proposed earlier (Van Deventer et al, 2017)

The cryo-EM map of CD9 in complex with EWI-F $F_{\triangle \mid g 1-5}$ and nanobody 4 C8 revealed a core CD9-EWI-F-EWI-F-CD9 heterotetramer arranged in a linear fashion. In this complex, nanobodies $4 \mathrm{C} 8$ bind to the CD9 on either side without contacting the EWI-F molecules. Both $C D 9_{\mathrm{EC} 2}$ segments are tilted by $\sim 40^{\circ}$ with respect to their TMD region (Fig $5 \mathrm{E}$ ), yielding an open conformation that is comparable with those predicted by MD simulation of CD81 and CD9 starting from the closed conformations observed in the crystal structures (Zimmerman et al, 2016; Umeda et al, 2020). CD9 predominantly contacts EWI-F through its TMD region, where CD9 helices h3 and h4 interact with the single-pass TM-helix of EWI-F (Fig 5D), which is in agreement with prior biochemical data that identified membrane helix $\mathrm{h} 4$ as a critical interface for binding EWI$F$ (André et al, 2009; Charrin et al, 2009b). The TMD regions of both CD9-EWI-F hetero-dimers are separated by more than $10-\AA$ distance and make no or minimal direct contacts. Instead, dimerization of the two hetero-dimers into a tetramer is dominated by contacts between the extracellular Ig6 domains of EWI- $F_{\Delta \mid g 1-5}$. Consistent with the absence of an intra-membrane interface between the two CD9-EWI-F heterodimers and no major extramembrane contacts between CD9 and EWI-F (Fig 5), the overall arrangement is highly flexible and we observed a range of lineararranged tetrameric complexes, ranging from an extended conformation to one that is bent by $\sim 50^{\circ}$ (Fig 6 and Video 1).

Most recently, Umeda et al (2020) reported a cryo-EM map of CD9 in complex with EWI-2 (Umeda et al, 2020). Overall, the structural arrangements of CD9-EWI-F $F_{\triangle I 1-5}$ and CD9-EWI-2 complexes are similar (Fig S5), in line with the partially overlapping cellular functions of EWI-F and EWI-2 (Stipp et al, 2001a; Sala-Valdés et al, 2006). As with CD9-EWI-F, major contacts are observed between h3 and h4 of CD9 and the single-pass TM helix of EWI-2. However, the membrane helices of EWI-2 are in closer proximity to each other than those of EWI-F, which could be correlated with the presence of two more putative palmitoylation sites in EWI-F than EWI-2. Furthermore, for CD9-EWI-2, only one conformation was reported, whereas the distinct shape of the $\mathrm{CD} 9_{\mathrm{EC} 2}-4 \mathrm{C} 8$ density enabled us to distinguish a range of straight to bent conformations for CD9-EWI- 

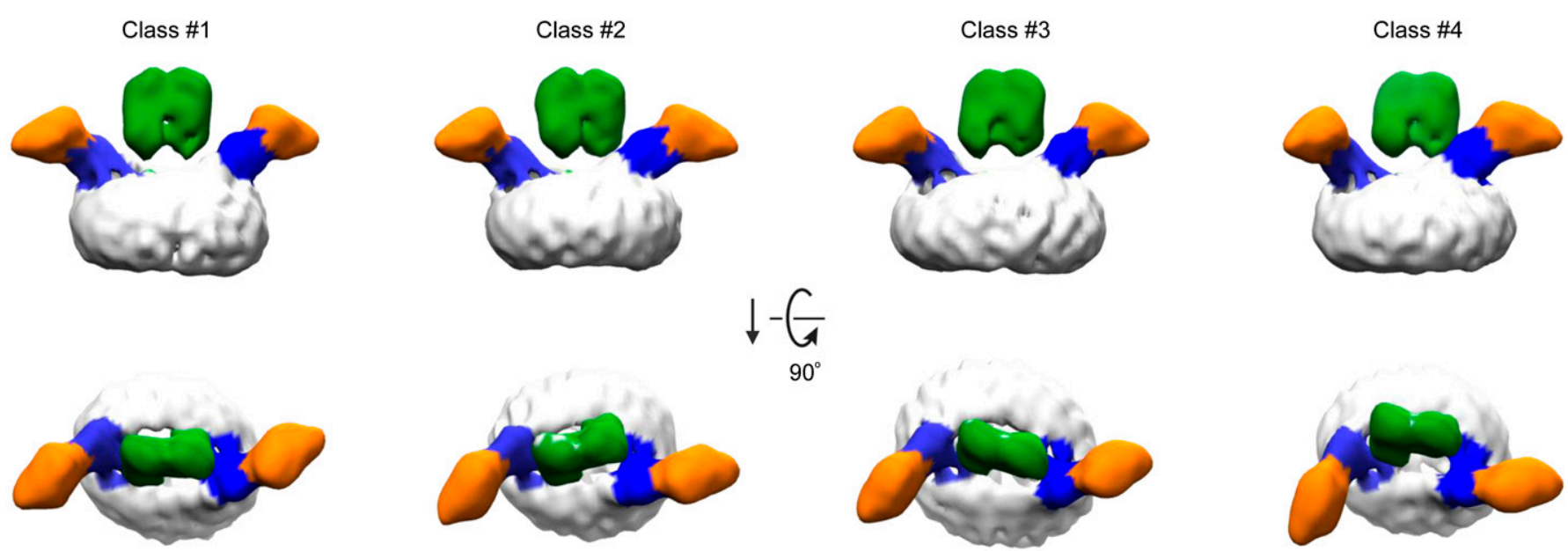

$90^{\circ}$
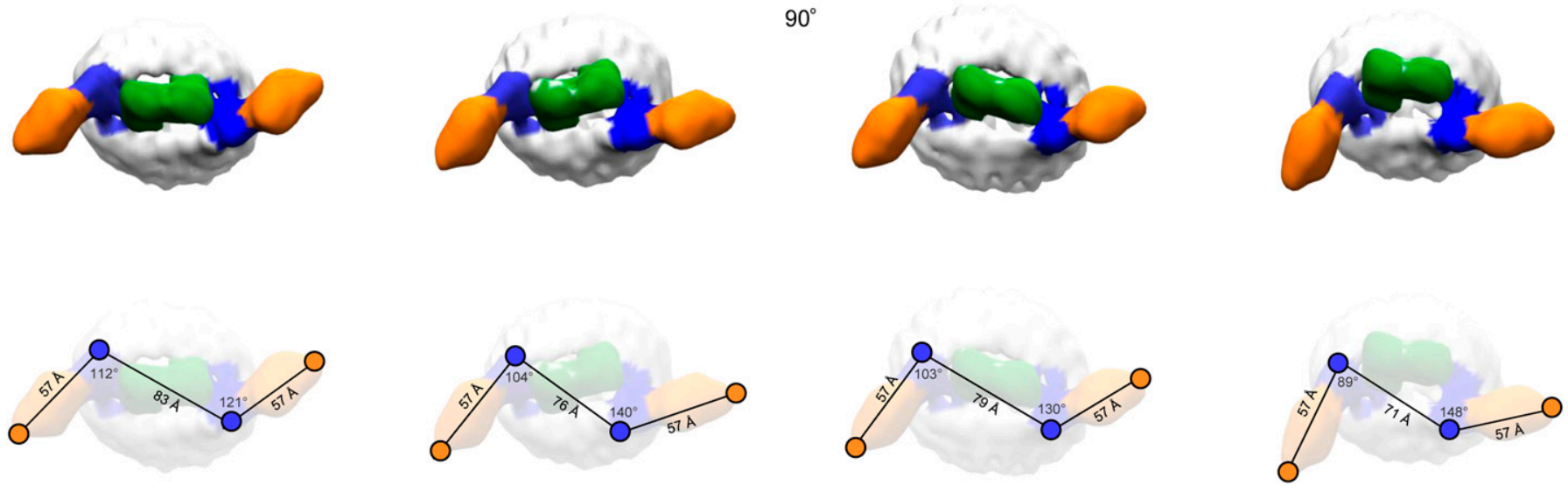

Figure 6. Flexibility of the EWI- $\mathrm{F}_{\Delta \mid \mathrm{g} 1-5}-\mathrm{CD} 9-4 \mathrm{C} 8$ revealed by 3D classifications.

Four classes obtained from a single 3D classification run are shown (see also Fig S4). The density regions corresponding to the Ig6-domain of EWI-F, CD9 EC2, $_{\text {, nanobody }}$ $4 \mathrm{C} 8$, and the digitonin micelle are colored green, blue, orange, and white, respectively. The top row depicts the four classes parallel to the membrane as a side view. The middle row shows the classes orthogonal to the membrane from the extracellular side. The bottom row depicts the density maps in the same orientation as in the middle row, with annotated distances and angles between CD9 ${ }_{\mathrm{EC} 2}$-residue K135 and 4C8-residue S121.

$\mathrm{F}_{\Delta \mid \mathrm{g} 1-5}$ (Fig 6 and Video 1). However, given the limited resolution of the cryo-EM maps of both cases, flexibility appears to be an inherent feature of the two complexes and a similar bending of CD9-EWI-2 cannot be excluded.

CD9, CD81, and other tetraspanins interact with various singlepass TM proteins that are known to homodimerize through their extra-membrane domains. Examples of homodimerizing partners of tetraspanins (besides EWI-F and EWI-2) include: DPP4 and ADAM17 for CD9; EGFR for CD82 (Odintsova et al, 2003); and heterodimeric integrins for CD151 (Berditchevski et al, 2002; Charrin et al, 2014, 2003). The observed linear-arranged tetramers of CD9-EWI-F and CD9-EWI-2 indicate that the interactions that govern complex formation are sequentially hydrophobic-hydrophilic-hydrophobic, with tetraspanin partner-protein interactions mediated by the TMDs on either side and protein partner homo-dimerization via their ectodomains in the center. Previous studies mapped a tetraspanin homo-dimerization site (for CD9) to membrane helices h1 and h2 (Kovalenko et al, 2005) and, for CD81, to the variable D-loop in the EC2 head domain. Both helices h1 and h2 and the D-loops are positioned on either end of the linear-arranged tetramers and orient outwards posed for putative interactions (Fig 5A). Thus, the observed hetero-tetrameric arrangements (Fig 6) suggest a simple "concatenation model" for forming transient higher order assemblies of end-to-end attached tetramers yielding small linear or circular structure (Fig 7A and B), which may explain the occurrence of TEMs and their highly dynamic nature. This single-particle cryoEM-derived model is in agreement with scanning EM data on immunogold-labeled CD81 and CD9 expressed on cells, which showed both tightly packed clusters and linear tetraspanin assemblies (Deneka et al, 2007; Grove et al, 2017). Thus, the concatenation model provides a structural basis for further studying the formation and signaling of TEMs in the plasma membrane.

\section{Materials and Methods}

\section{Chemicals}

All chemicals were purchased from Sigma-Aldrich unless specified otherwise.

\section{Construct design}

The CDNA encoding for CD9 and EWI-F was obtained as described previously (Neviani et al, 2020). Briefly, codon-optimized cDNA for mammalian cell expression, encoding for human CD9 was purchased from GeneArt. Full-length CD9 was cloned in a pUPE expression vector (U-Protein Express BV) with either a $\mathrm{N}$-terminal Strep-II tag or a N-terminal Strep3-GFP tag with a TEV-protease site. The CD9-W6 construct (CD9-C9W, C78W, C79W, C87W, C218W, and C219W), with all palmitoylated cysteines mutated to tryptophan, was cloned as described (Neviani et al, 2020). The construct encoding for the EC2 domain of CD9 (CD9 ${ }_{\mathrm{EC} 2}$, residues K114-N191) was generated 
A

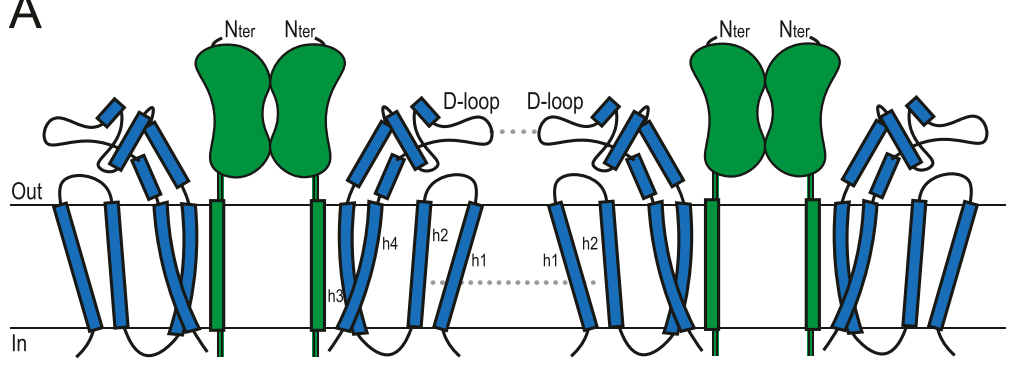

B

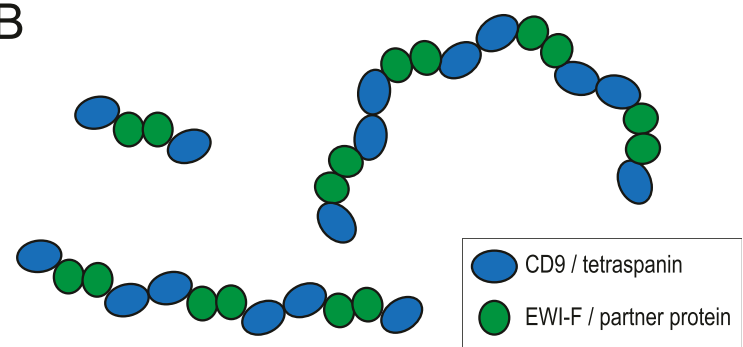

Figure 7. Concatenation model of tetraspanin-enriched microdomain formation.

(A) Cartoon model for the formation of higher order oligomers based on the hetero-tetrameric CD9-EWI-F arrangement observed in the cryo-EM data and biochemical interaction studies. (A, B) Top-view model, shown orthogonal to the membrane, of putative linear and circular tetraspanin-enriched imicrodomain assemblies based the straight and bent conformations adopted by the CD9-EWI-F complex and the oligomerization model in panel (A).

with a PCR reaction using the Q5-PCR kit (NEB). The $\mathrm{CD}_{\mathrm{EC} 2}$ construct was cloned in a pUPE expression vector with an N-terminal cystatin signal peptide and a C-terminal 6x-His tag. cDNA encoding for fulllength human EWI-F was obtained from Source BioScience. EWI-F truncations were generated using PCR. Construct boundaries of EWI$F_{\triangle I g 1}(H 146-D 879), E W I-F_{\Delta I g 1-2}(Q 275-D 879), E W I-F_{\Delta I g 1-3}(E 405-D 879)$, EWI-F $F_{\triangle I \mathrm{~g} 1-4}(\mathrm{~N} 540-\mathrm{D} 879)$, and $E W I-F_{\triangle \mid \mathrm{g} 1-5}$ (T685-D879) were based on Uniprot domain assignments (UniprotKB-Q9P2B2). All EWI-F constructs were cloned in a pUPE expression vector with an N-terminal cystatin signal peptide and a C-terminal $6 x-H i s$ tag.

\section{Nanobody selection and production}

Phage library construction and anti-CD9 nanobody selections were carried out as described before and resulted in the selection of two nanobodies: 4C8 and 4E8 (Neviani et al, 2020). DNA sequences of clones 4C8 and 4E8 were ligated into a modified pHEN6 vector with a C-terminal thrombin-cleavable 6 xHis tag and an $\mathrm{N}$-terminal pelB leader sequence for periplasmic secretion (gift from dr. F Opazo, University of Göttingen Medical Center, Göttingen). For large-scale nanobody production, Escherichia coli BL-21 Codon Plus (DE3)-RIL bacteria (Agilent Technologies Inc.), transformed with nanobodyencoding plasmids, were batch cultured using a BioFlo/CelliGen 115 bioreactor (New Brunswick Scientific). Overnight bacteria cultures in LB, 2\% (wt/vol) glucose, $100 \mu \mathrm{g} / \mathrm{ml}$ ampicillin, $35 \mu \mathrm{g} / \mathrm{ml}$ chloramphenicol were inoculated (1:100 dilution) in 5 liters of terrific broth medium (24 g/l yeast extract, $20 \mathrm{~g} / \mathrm{l}$ tryptone, $4 \mathrm{ml} / \mathrm{l}$ glycerol, $0.017 \mathrm{M} \mathrm{KH}_{2} \mathrm{PO}_{4}$, and $0.072 \mathrm{M} \mathrm{K}_{2} \mathrm{HPO}_{4}$ ) supplemented with $0.1 \%$ (wt/ vol) glucose, $100 \mu \mathrm{g} / \mathrm{ml}$ ampicillin, $0.01 \%$ (vol/vol) antifoam 204 and allowed to grow at $37^{\circ} \mathrm{C}$, while maintaining the dissolved oxygen of the medium at $70 \%$. When optical density at $600 \mathrm{~nm}$ was around 1-2, isopropyl-h-D-thiogalactopyranoside (Thermo Fisher Scientific) was added to the culture at a final concentration of $1 \mathrm{mM}$, to induce protein production. Fermentation was continued overnight at $25^{\circ} \mathrm{C}$. The next day, bacterial biomass was harvested by centrifugation at $5,400 \mathrm{~g}$ and subsequently resuspended in PBS. The bacterial suspension was subjected to two cycles of freezing at $-20^{\circ} \mathrm{C}$ and thawing, followed by a centrifugation at $27,000 \mathrm{~g}$. The supernatant was collected and His-tagged nanobodies were purified using HisTrap column chromatography (GE Healthcare) followed by a buffer exchange step to PBS using a HiTrap desalting column (GE Healthcare). Nanobodies were stored in PBS at $-20^{\circ} \mathrm{C}$ until further use. Nanobody 4C8 was treated with Thrombin (0.5 U/100 $\mu \mathrm{g}$; GE Healthcare) for $16 \mathrm{~h}$ at $22^{\circ} \mathrm{C}$, to remove the $6 \mathrm{xHis}$ purification tag. $4 \mathrm{C} 8$ was then injected into a Superdex75 10/300 column (GE Healthcare) pre-equilibrated in buffer A ( 25 mM Hepes, pH 7.5, 150 $\mathrm{mM} \mathrm{NaCl}$ ), and monodisperse fractions were collected. The tag of nanobody 4 E8 was not removed before crystallization.

\section{Determination of nanobody binding affinity}

The binding affinity of 4C8 and 4E8 on CD9 was determined both on purified CD9 and on CD9 endogenously expressed on HeLa cells as described in detail (Neviani et al, 2020). Binding was carried on either 100 ng wtCD9-3Strep captured on Strep-Tactin coated wells; or HeLa cells seeded in 96-well plates. On the day of the assay, the target was incubated with serial dilutions of nanobodies for $2 \mathrm{~h}$ either in $2 \%$ (wt/vol) BSA in buffer A supplemented with $0.025 \%$ (wt) vol) $\mathrm{N}$-dodecyl- $\beta$-D-maltoside (DDM) at rt in the case of purified protein or in binding buffer (1\% wt/vol BSA and 25 mM Hepes, pH 7.2, in DMEM without phenol red; Lonza Netherlands B.V.) at $4^{\circ} \mathrm{C}$ in the case of HeLa cells, in triplicate. Nanobodies were detected after incubation with rabbit anti- $\mathrm{V}_{\mathrm{H}} \mathrm{H}$ antibody (1:1,000 in $2 \% \mathrm{wt} / \mathrm{vol} \mathrm{BSA}$ in PBS; clone k1216; QVQ B.V.) for $1 \mathrm{~h}$ at room temperature, followed by 1-h incubation with goat anti-rabbit IRDye800CW (1:1,000 in 2\% wt/vol BSA in PBS; LI-COR Biosciences) at rt. For cell binding assays, the cells were fixed with $4 \%$ formaldehyde before incubation with the detecting antibodies. Finally, IRDye800CW fluorescent signal at $800 \mathrm{~nm}$ was detected with Odyssey Infrared Imager (LI-COR Biosciences). The measured fluorescent intensities were normalized, setting as $100 \%$ the mean of intensities detected at the higher nanobody concentrations, and plotted (mean \pm SEM) over protein concentration using GraphPad Prism 7. A nonlinear regression curve for one-site specific binding was fitted to determine apparent binding affinity $\left(K_{D}\right)$ of the different nanobodies.

\section{$\mathrm{CD}_{\mathrm{EC} 2}$ expression and purification}

CD9 ${ }_{\mathrm{EC} 2}$ was recombinantly expressed in HEK293-EBNA cells (provided by U-Protein Express BV). Cells were grown at $37^{\circ} \mathrm{C}$ and harvested after $6 \mathrm{~d}$. The cells were centrifuged at 1,000 $\mathrm{g}$ for $10 \mathrm{~min}$, after which the medium was collected. Two different purification strategies were then used: 
1) For the protein sample used for the crystallization of $C D 9_{E C 2}$ in the absence and presence of nanobody $4 \mathrm{C} 8$, the medium was loaded overnight onto a 5-ml anti-CD9 AR40A746.2.3 affinity column (7.5 mg antibody/ml CNBr Activated Sepharose 4B beads; GE Healthcare) in line of an AKTA explorer (GE Healthcare). After loading, unbound proteins were washed away, and bound $\mathrm{CD} 9_{\mathrm{E}, \mathrm{C} 2}$ was eluted using 0.2 $\mathrm{M}$ glycine, $150 \mathrm{mM} \mathrm{NaCl}, \mathrm{pH}$ 2.5, and neutralized with 1:10 volume of $1 \mathrm{M}$ Tris pH 9.0. A size-exclusion step on a Superdex 200 10/300 Increase column equilibrated in buffer $\mathrm{A}$ ( $25 \mathrm{mM}$ Hepes, pH 7.5, $150 \mathrm{mM} \mathrm{NaCl}$ ) was performed. Next, another affinity-purification step was performed on a $5 \mathrm{ml}$ anti-CD9 AT1412dm (Neviani, 2019) affinity column (7.5 mg antibody/ml CNBr Activated Sepharose 4B beads). After loading, unbound proteins were washed away and bound $\mathrm{CD}_{\mathrm{EC} 2}$ was eluted with $0.2 \mathrm{M}$ Glycine, $150 \mathrm{mM} \mathrm{NaCl} \mathrm{pH} 2.5$ and neutralized 1:10 volume of $1 \mathrm{M}$ Tris $\mathrm{pH}$ 9.0. A final size-exclusion step on a Superdex 200 10/300 Increase column equilibrated in buffer $A$ was performed before crystallization.

2) For the protein sample used for the crystallization of $C D 9_{E C 2}$ in complex with nanobody $4 \mathrm{E} 8$, the cell medium was incubated with $\mathrm{Ni}$-Sepharose Excel beads (GE Healthcare) at $4^{\circ} \mathrm{C}$ for $2 \mathrm{~h}$. The beads were washed for 10 column volumes in buffer $\mathrm{B}(50 \mathrm{mM}$, Tris $\mathrm{pH} 7.8$, $500 \mathrm{mM} \mathrm{NaCl}$ ) with $10 \mathrm{mM}$ imidazole and $\mathrm{CD} 9_{\mathrm{EC} 2}$ was subsequently eluted from the beads with $300 \mathrm{mM}$ imidazole in buffer $\mathrm{B}$. The protein sample was then concentrated and incubated with $2 \mathrm{mM}$ DTT (final concentration) for $\sim 16 \mathrm{~h}$ to remove intermolecular, nonnative disulfide bonds. $C D 9_{\mathrm{EC} 2}$ was then injected on a Superdex75 $10 / 300$ column (GE Healthcare) pre-equilibrated in buffer C (20 mM Tris $\mathrm{pH} \mathrm{8,} 150 \mathrm{mM} \mathrm{NaCl}$ ). Monomeric protein fractions were pooled based on non-reducing SDS-PAGE. To form a complex with nanobody $4 \mathrm{E} 8,230 \mu \mathrm{l}$ of $\mathrm{CD} 9_{\mathrm{EC} 2}$ at $5 \mathrm{mg} / \mathrm{ml}$ was mixed with $250 \mu \mathrm{l}$ of $4 \mathrm{E} 8(7 \mathrm{mg} / \mathrm{ml}$, in PBS) and incubated for $30 \mathrm{~min}$. The mixture was injected on a Superdex75 10/300 column pre-equilibrated in buffer $\mathrm{C}$ and fractions containing both $\mathrm{CD} 9_{\mathrm{EC} 2}$ and $4 \mathrm{E} 8$ were collected and concentrated to $\sim 8.0 \mathrm{mg} / \mathrm{ml}$.

\section{Crystallization and data collection}

$\mathrm{CD} 9_{\mathrm{EC} 2}(10 \mathrm{mg} / \mathrm{ml})$ was crystallized in sitting drop in 33\% wt/vol pentaerythritol propoxylate, $0.2 \mathrm{M} \mathrm{KCl}$, and $0.1 \mathrm{M}$ sodium citrate at $\mathrm{pH}$ 6.0. No further cryoprotectant was used.

For the CD9EC2-4C8 complex, CD9 ${ }_{\mathrm{EC} 2}(12 \mathrm{mg} / \mathrm{ml}$ in buffer $\mathrm{A}$ ) was mixed with 4 C8 $(5.9 \mathrm{mg} / \mathrm{ml}$ in buffer $\mathrm{A})$ in a 1:1 molar ratio. Crystals grew in sitting drop in 0.095 M sodium citrate, pH 5.6, $5 \%$ (vol/vol) glycerol, 19\% (vol/vol) isopropanol, 20\% (wt/vol) PEG 4,000. Crystals were cryoprotected by soaking in reservoir solution supplemented with $25 \%$ glycerol (final concentration).

The $\mathrm{CD} 9_{\mathrm{EC} 2}-4 \mathrm{E} 8$ complex was purified as described above and concentrated to $8.0 \mathrm{mg} / \mathrm{ml}$. Crystals grew in hanging drop in $0.2 \mathrm{M}$ sodium acetate, $0.1 \mathrm{M}$ Tris, pH 8.0, 30\% (wt/vol) PEG 4,000 and were cryoprotected by soaking in reservoir solution supplemented with 20\% (vol/vol) ethylene glycol.

For the crystallization of nanobody $4 \mathrm{C} 8$ alone $(5.1 \mathrm{mg} / \mathrm{ml})$, crystals grew in sitting drop in $0.1 \mathrm{M}$ Hepes, pH 7.5, 20\% (wt/vol) PEG 8,000 and were cryoprotected by soaking in reservoir solution supplemented with 30\% (vol/vol) PEG 400.

All crystals were flash frozen in liquid nitrogen immediately after harvesting. Diffraction data were collected at Diamond Light Source on beamlines I-03 (CD9 $\left.\mathrm{EC2}_{2}-4 \mathrm{C} 8\right), \mathrm{I}-04\left(\mathrm{CD} 9_{\mathrm{EC} 2}\right)$ and I-04-1 (CD9 $\left.{ }_{\mathrm{EC2}}-4 \mathrm{E} 8\right)$, or at the European Synchrotron Radiation Facility on beamline ID23-2 (4C8 alone).

\section{$\mathrm{CD} 9_{\mathrm{EC} 2}$ crystal data processing and refinement}

The triclinic $\mathrm{CD} 9_{\mathrm{EC} 2}$ crystal was twinned with a twofold rotation about $a^{*}+b^{*}$ as the twinning operation. Consequently, two orientation matrices were used for integration with the Eval15 software (Schreurs et $\mathrm{al}, 2010)$. In the prediction of reflection profiles, an isotropic mosaicity of $1.1^{\circ}$ and a mica expansion of 0.077 along $a^{*}+b^{*}$ was assumed. The resulting reflection file contained $21.5 \%$ overlapping reflections belonging to both twin domains. Initial de-twinning was performed with the TWINABS software (Sheldrick, 2009). These data were used for structure solution by molecular replacement, using PHASER (McCoy et al, 2007) within the CCP4 software suite (Potterton et al, 2018), using the model of $C D 9_{E C 2}$ from the previously solved

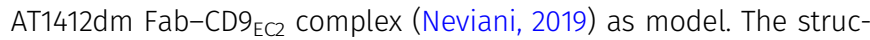
ture was iteratively refined using Refmac5 (Murshudov et al, 2011) alternated with model improvement in COOT (Emsley \& Cowtan, 2004). Local non-crystallographic symmetry restraints were maintained during refinement. The calculated structure factors from this refinement were then used for the final de-twinning of the overlapping data. The scale factor of $6.2147 \times \exp \left(-8.19544 \times \sin (\theta / \lambda)^{2}\right)$ between the two twin domains was determined with XPREP (BrukerAXS, 2008), based on the non-overlapping reflections. Interframe scaling of the de-twinned data was performed with SADABS and merging was performed with the CCP4 suite. Final refinement rounds in Refmac5 using the latest data yielded $R_{\text {work }} / R_{\text {free }}=23.9 / 27.9 \%$ and the structure was deposited in the RCSB Protein Data Bank under accession code 6RLR.

\section{$\mathrm{CD} 9_{\mathrm{EC} 2}-4 \mathrm{C} 8$ crystal data processing and refinement}

Diffraction images were processed using DIALS (Winter et al, 2018) and the integrated reflection data were truncated anisotropically using the STARANISO web server (Tickle et al, 2018). The structure was solved by molecular replacement using PHASER with the $\mathrm{CD} 9_{\mathrm{EC} 2}$ structure and a nanobody homology model obtained through the SWISS-MODEL server (Waterhouse et al, 2018) as search models. The structure was iteratively refined using Refmac5 or Phenix (Adams et al, 2002) alternated with manual model improvement in COOT. The final refinement in Phenix yielded $R_{\text {work }} / R_{\text {free }}=20.6 / 24.8 \%$ and the structure was deposited in the RCSB Protein Data Bank under accession code $6 Z 20$.

\section{C8 crystal data processing and refinement}

Diffraction images were processed using Eval15 (Schreurs et al, 2010). The structure was solved by molecular replacement using PHASER with the nanobody in chain B of PDB 5IMK as the search model. The nanobody residues were manually adjusted to the 4C8 amino-acid sequence in Coot. The structure was then iteratively refined using Refmac5 or Phenix, alternated with manual model improvement in COOT. The final refinement in Phenix yielded $R_{\text {work }} /$ $R_{\text {free }}=20.1 / 23.1 \%$ and the structure was deposited in the RCSB Protein Data Bank under accession code 6Z1Z. 


\section{CD9 ${ }_{\mathrm{EC} 2}-4 \mathrm{E} 8$ crystal data processing and refinement}

For the $\mathrm{CD} 9_{\mathrm{EC} 2}-4 \mathrm{E} 8$ dataset, the autoprocessed and anisotropicaltruncated (autoPROC-STARANISO) reflection data file provided by Diamond Light Source was used. The structure was solved by molecular replacement using PHASER with the $\mathrm{CD} 9_{\mathrm{EC} 2}-4 \mathrm{C} 8$ structure as search model. The $4 \mathrm{C} 8$ residues were replaced with the corresponding 4E8 residues and the CDR regions of the nanobody were manually built in Coot. The structure was then iteratively refined using Refmac5 or Phenix, alternated with model improvement in COOT. The final refinement in Phenix yielded $R_{\text {work }} / R_{\text {free }}=15.1 / 19.0 \%$ and the structure was deposited in the RCSB Protein Data Bank under accession code 6Z1V.

\section{Large-scale expression and purification of CD9 and full-length EWI-F}

N-Strep3-GFP-tagged CD9-W6 and full-length EWI-F were transiently expressed in 2 liters Epstein-Barr virus nuclear antigen I (EBNA1)-expressing HEK293 cell cultures (HEK293-EBNA, provided by U-Protein Express BV). The cells were grown at $37^{\circ} \mathrm{C}$ and harvested after $4 \mathrm{~d}$. All subsequent steps were carried out at $4^{\circ} \mathrm{C}$. The cells were washed in PBS and then lysed in buffer containing $50 \mathrm{mM}$ Tris, pH 7.8, $150 \mathrm{mM} \mathrm{NaCl}, 1 \%$ (wt/vol) N-dodecyl- $\beta$-D-maltoside (DDM; Anatrace), and protease inhibitor cocktail (Roche) for $2 \mathrm{~h}$. The lysed sample was ultracentrifuged for $45 \mathrm{~min}$ at 100,000 $\mathrm{g}$ to remove insoluble membranes and cell debris. The supernatant was incubated with Strep-Tactin resin (GE Healthcare) for $2 \mathrm{~h}$, and the resin was washed with 20 column volumes of buffer D ( 50 mM Tris, pH 7.8, 150 mM $\mathrm{NaCl}$, and $0.025 \%$ [wt/vol] DDM). Protein was eluted from the resin with buffer D supplemented with $3.5 \mathrm{mM}$ desthiobiotin. The eluted fractions were pooled, concentrated in a 100-kD concentration device (Amicon), and injected on a Superose6 10/300 increase column (GE Healthcare) pre-equilibrated in buffer D. Fractions containing both CD9 and EWI-F were pooled and concentrated to $\sim 3.3 \mathrm{mg} / \mathrm{ml}$.

\section{Small-scale expression and purification of CD9 with EWI-F variants}

N-Strep3-GFP-tagged CD9-W6 was co-transfected with EWI-F, EWI-F ${ }_{\triangle I \mathrm{gl}}$,

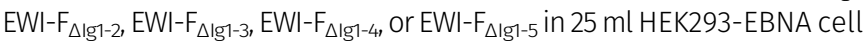
cultures (provided by U-Protein Express BV). Cells were grown at $37^{\circ} \mathrm{C}$ and harvested after $4 \mathrm{~d}$. Cells were washed in PBS and then lysed in buffer containing $50 \mathrm{mM}$ Tris, $\mathrm{pH} 7.8,150 \mathrm{mM} \mathrm{NaCl}, 1 \%$ (wt/ vol) DDM, 0.5\% (wt/vol) digitonin (Calbiochem), and protease inhibitor cocktail (Roche) for $2 \mathrm{~h}$. The supernatant was incubated with Strep-Tactin resin (GE Healthcare) for $2 \mathrm{~h}$, and the resin was washed with buffer $\mathrm{E}$ (50 mM Tris pH, 7.8, $150 \mathrm{mM} \mathrm{NaCl}$, and $0.08 \%$ [wt/vol] digitonin) in spin columns. Protein was eluted from the resin with buffer E supplemented with $3.5 \mathrm{mM}$ desthiobiotin. Complex formation between CD9 and the co-transfected EWI-F variants was assessed by SDS-PAGE.

\section{Large-scale expression and purification of CD9 and EWI-F $F_{\Delta I g 1-5}$ with nanobody $4 \mathrm{C} 8$}

$\mathrm{N}$-Strepll-tagged, wild-type $\mathrm{CD} 9$, and $\mathrm{EWI}-\mathrm{F}_{\triangle \mid \mathrm{Ig} 1-5}$ were expressed in 3 liters HEK293-EBNA GNT1-cell cultures. The cells were grown at $37^{\circ} \mathrm{C}$ and harvested after $4 \mathrm{~d}$. All subsequent steps were carried out at $4^{\circ} \mathrm{C}$. The cells were washed in PBS and then lysed in buffer containing 50 $\mathrm{mM}$ Tris, $\mathrm{pH} 7.8,150 \mathrm{mM} \mathrm{NaCl}, 1 \%$ (wt/vol) digitonin, 0.2\% (wt/vol) DDM and protease inhibitor cocktail (Roche) for $2 \mathrm{~h}$. The lysed sample was ultracentrifuged for $45 \mathrm{~min}$ at 100,000g to remove insoluble membranes and cell debris. The supernatant was incubated with StrepTactin resin (GE Healthcare) for $2 \mathrm{~h}$, and the resin was washed with 20 column volumes of buffer $\mathrm{E}$ ( $50 \mathrm{mM}$ Tris, pH 7.8, $150 \mathrm{mM} \mathrm{NaCl}, 0.08 \%$ [wt/vol] digitonin). Protein was eluted from the resin with buffer $E$ supplemented with $3.5 \mathrm{mM}$ desthiobiotin. The eluted fractions were pooled, concentrated in a $100-\mathrm{kD}$ concentration device (Amicon) to $\sim 1.5 \mathrm{mg} / \mathrm{ml}$, and incubated with $\mathrm{EndoH}_{\mathrm{F}}$ (NEB) at a volume to volume ratio of 1:20 for $2 \mathrm{~h}$ to remove the $\mathrm{N}$-linked glycan of EWI- $\mathrm{F}_{\triangle I \mathrm{~g} 1-5}$. The complex was then incubated with a large excess of nanobody $4 \mathrm{C} 8$ (in PBS buffer with $0.08 \%$ [wt/vol] digitonin) and injected on a Superdex 200 10/300 increase column (GE Healthcare) pre-equilibrated in buffer E. Fractions containing the EWI- $\mathrm{F}_{\Delta \mid \mathrm{g} 1-5}-\mathrm{CD} 9-4 \mathrm{C} 8$ complex were pooled and concentrated to $\sim 3 \mathrm{mg} / \mathrm{ml}$.

\section{Cryo-EM grid preparation and data collection}

$2.8 \mu \mathrm{l}$ of CD9-EWI-F Full-Length $(3.3 \mathrm{mg} / \mathrm{ml})$ or EWI-F $\mathrm{F}_{\Delta \mathrm{g} 1-5}-\mathrm{CD} 9-4 \mathrm{C} 8$ (3 $\mathrm{mg} / \mathrm{ml}$ ) was pipetted onto a glow-discharged R1.2/1.3 200 mesh Au holey carbon grid and then plunge-frozen in a liquid ethane/ propane mixture using a Vitrobot Mark IV (Thermo Fisher Scientific). The blotting was performed at $20^{\circ} \mathrm{C}$ for $4 \mathrm{~S}$ with blot force 0 (for

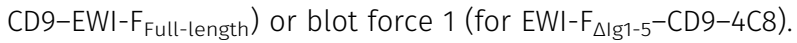

All data were collected on a 200-kV Talos Arctica microscope (Thermo Fisher Scientific) equipped with a K2-summit detector (Gatan) and a post-column $20 \mathrm{keV}$ energy filter, using EPU automated-data collection software (Thermo Fisher Scientific). Movies for the CD9-EWI-F Full-Length dataset were collected in super-resolution mode (binned pixel size 1.03 $\AA$ ) in 28 frames for $7 \mathrm{~s}$, with an electron exposure of $1.82 \mathrm{e}^{-} / \AA^{2} /$ frame (total exposure $50.9 \mathrm{e}^{-} / \AA^{2}$ ). Movies for the EWI-F $\mathrm{F}_{\Delta \mathrm{g} 1-5}-\mathrm{CD} 9-4 \mathrm{C} 8$ dataset were collected in counting mode (pixel size $1.03 \AA$ ) in 36 frames for $7.2 \mathrm{~s}$, with an electron exposure of $1.45 \mathrm{e}^{-} / \AA^{2} /$ frame (total exposure $52.0 \mathrm{e}^{-} / \AA^{\circ}$ ).

\section{Cryo-EM image processing}

For the CD9-EWI-F Full-Length dataset, 379 micrographs were imported in the RELION 3.0beta pipeline (Scheres, 2012). The super-resolution mode recorded micrographs were binned $2 x$, gain corrected and motion corrected using MotionCor2 (Zheng et al, 2017), and GCTF (Zhang, 2016) was used to estimate the contrast transfer function (CTF) for each micrograph. 880 particles were manually picked and 2D classified. The resulting 2D-class averages were then used as templates for automated particle picking in RELION (Scheres, 2015), yielding 29,216 particles, which were binned $2 \times$ upon particle extraction. These particles were subjected to numerous 2D classification runs. Further processing was not attempted due to strong heterogeneity in the particles.

For the EWI- $\mathrm{F}_{\triangle \mid \mathrm{g} 1-5}-\mathrm{CD} 9-4 \mathrm{C} 8$ dataset, 10,724 micrographs were imported in the RELION 3.1beta pipeline. The micrographs were motion-corrected and gain-corrected using MotionCor2, and GCTF was used to estimate the CTF for each micrograph. 428 micrographs were discarded based on poor CTF estimations, yielding a total of 10,296 micrographs for further processing. The motion-corrected micrographs were imported in EMAN2 (Tang et al, 2007), and several 
hundred good particles and bad particles, as well as background images, were picked manually for training of the EMAN2 NeuralNet particle picker, which was subsequently used for automated particle picking. The obtained particle coordinates were imported back into the RELION pipeline and 1,148,718 particles were extracted and binned 3× (resulting pixel size $3.09 \AA$ ). The particles were 3D classified into four classes, and the 107,830 particles belonging to one obvious junk class were discarded. 1,040,888 particles were then subjected to two rounds of 2D classification into 200 classes, through which 352,979 particles were removed. The remaining 687,909 particles were $3 \mathrm{D}$ classified into 20 classes. The highest-populated class, comprising 354,272 particles, was 3D-auto refined using a mask and solvent flattening Fourier shell correlations (FSCS), which yielded a map at a global resolution of $8.6 \AA$ based on the gold-standard FSC $=0.143$ criterion (Rosenthal \& Henderson, 2003). This map was sharpened with a B-factor of $-1,200 \AA^{2}$ and filtered based on local-resolution in RELION. Another 3D classification with the 687,909 particles (following the 2D classifications) was performed into five classes, while ignoring the CTFs until the first peak, meaning that CTF-amplitude correction was only performed from the first peak of each CTF onward. This strategy resulted in better particle separation in distinct classes. However, further subclassifications, either with or without regions of the protein complex masked out, yielded density maps of worse quality and resolution. This suggested a continuous disorder in the protein complex rather than that few discrete conformations were adopted by EWI- $F_{\triangle I g 1-5}-\mathrm{CD} 9-4 \mathrm{C} 8$. The particles were not un-binned as the Nyquist frequency ( $6.2 \AA$ ) was not reached in any refinement.

\section{Modeling in cryo-EM maps}

Protein models were rigid-body fitted into the sharpened $8.6-\AA$

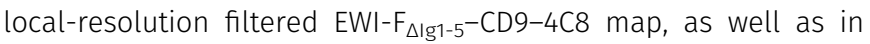
non-sharpened maps obtained through 3D classifications, using the "Fit in Map" option in UCSF Chimera (Goddard et al, 2007).

\section{Figure preparation}

The crystal-structure figures were prepared using Pymol (Schrödinger). All figures containing cryo-EM density maps were generated using UCSF Chimera. The morph between four observed conformations of the EWI-F ${ }_{\triangle I 91-5}-$ CD9-4C8 complex (Video 1) was made in UCSF Chimera and edited using Adobe Premiere Project. The cartoon models (Fig 7) were prepared in Adobe Illustrator.

\section{Data Availability}

Data supporting the findings of this manuscript are available from the corresponding author (P Gros) upon reasonable request. The relevant cryo-EM density maps of the EWI- $\mathrm{F}_{\triangle \mid \mathrm{g} 1-5}-\mathrm{CD} 9-4 \mathrm{C} 8$ dataset have been deposited in the Electron Microscopy Data Bank under accession number EMDB-11053. This deposition comprises the sharpened, localresolution filtered map, unfiltered-half maps, and four 3D-class averages. Model coordinates of the crystal structures have been deposited in the Protein Data Bank under accession numbers 6RLR $\left(\mathrm{CD} 9_{\mathrm{EC} 2}\right), 6 Z 20\left(\mathrm{CD} 9_{\mathrm{EC} 2}-4 \mathrm{C8}\right), 6 \mathrm{Z1V}\left(\mathrm{CD9}_{\mathrm{EC} 2}-4 \mathrm{E} 8\right)$, and $6 \mathrm{Z1Z}$ (4C8).

\section{Supplementary Information}

Supplementary Information is available at https://doi.org/10.26508/lsa 202000883.

\section{Acknowledgements}

We thank the beamline scientists of Diamond Light Source and the European Synchrotron Radiation Facility for assistance during data collection; we acknowledge W Hemrika (U-Protein Express BV) for mammalian cell cultures; we thank SC Howes, CTWM Schneijdenberg, and JD Meeldijk of the Utrecht EMsquare for electron-microscope assistance and maintenance; we thank D El Mazouni for fruitful discussions; we are grateful to $A B$ van Spriel and $S$ van Deventer of Radboud University Medical Center Nijmegen for proofreading of the manuscript. This work has been supported by The Netherlands Organization for Scientific Research (NWO), Fund Nieuwe Chemische Innovaties (NCI) Technology Area (project no. 731.015.201) to PMP van Bergen en Henegouwen and P Gros; and the Institute of Chemical Immunology (project 024.002.009) to P Gros.

\section{Author Contributions}

W Oosterheert: conceptualization, data curation, formal analysis, validation, investigation, visualization, methodology, and writing-original draft, review, and editing.

KT Xenaki: data curation, formal analysis, investigation, methodology, and writing-review and editing.

$\checkmark$ Neviani: data curation, investigation, methodology, and writing-review and editing.

W Pos: investigation and methodology.

S Doulkeridou: investigation and methodology.

J Manshande: investigation and methodology.

NM Pearce: validation, investigation, methodology, and writing-review and editing.

LMJ Kroon-Batenburg: validation, investigation, and methodology. M Lutz: validation, investigation, and methodology.

PMP Van Bergen en Henegouwen: formal analysis, supervision, funding acquisition, validation, and writing-review and editing. P Gros: conceptualization, formal analysis, supervision, funding acquisition, validation, and writing-original draft, review, and editing.

\section{Conflict of Interest Statement}

The authors declare that they have no conflict of interest.

\section{References}

Adams PD, Grosse-Kunstleve RW, Hung L-W, loerger TR, McCoy AJ, Moriarty NW, Read RJ, Sacchettini JC, Sauter NK, Terwilliger TC (2002) PHENIX: Building new software for automated crystallographic structure determination. Acta Crystallogr D Biol Crystallogr 58: 1948-1954. doi:10.1107/S0907444902016657

André M, Chambrion C, Charrin S, Soave S, Chaker J, Boucheix C, Rubinstein E, Le Naour F (2009) In situ chemical cross-linking on living cells reveals CD9P-1 cis-oligomer at cell surface. J Proteomics 73: 93-102. doi:10.1016/j.jprot.2009.08.005

André M, Morelle W, Planchon S, Milhiet PE, Rubinstein E, Mollicone R, Chamot-Rooke J, Le Naour F (2007) Glycosylation status of the 
membrane protein CD9P-1. Proteomics 7: 3880-3895. doi:10.1002/ pmic.200700355

Andreu Z, Yáñez-Mó M (2014) Tetraspanins in extracellular vesicle formation and function. Front Immunol 5: 1-12. doi:10.3389/fimmu.2014.00442

Bennett MJ, Choe S, Eisenberg D (1994) Domain swapping: Entangling alliances between proteins. Proc Natl Acad Sci U S A 91: 3127-3131. doi:10.1073/pnas.91.8.3127

Berditchevski F, Odintsova E, Sawada S, Gilbert E (2002) Expression of the palmitoylation-deficient CD151 weakens the association of $\alpha 3 \beta 1$ integrin with the tetraspanin-enriched microdomains and affects integrin-dependent signaling. J Biol Chem 277: 36991-37000. doi:10.1074/jbc.M205265200

Berditchevski F, Zutter MM, Hemler ME (1996) Characterization of novel complexes on the cell surface between integrins and proteins with 4 transmembrane domains (TM4 proteins). Mol Biol Cell 7: 193-207. doi:10.1091/mbc.7.2.193

Boucheix C (2000) Severely reduced female fertility in CD9-deficient mice. Science 287: 319-321. doi:10.1126/science.287.5451.319

Brosseau C, Colas L, Magnan A, Brouard S (2018) CD9 tetraspanin: A new pathway for the regulation of inflammation? Front Immunol 9: 1-12. doi:10.3389/fimmu.2018.02316

Bruker-AXS (2008) XPREP: Data preparation and reciprocal space exploration

Chambrion C, le Naour F (2010) The tetraspanins CD9 and CD81 regulate CD9P1-induced effects on cell migration. PLoS One 5: 1-12. doi:10.1371/ journal.pone.0011219

Charrin S, Jouannet S, Boucheix C, Rubinstein E (2014) Tetraspanins at a glance. J Cell Sci 127: 3641-3648. doi:10.1242/jcs.154906

Charrin S, Latil M, Soave S, Polesskaya A, Chrétien F, Boucheix C, Rubinstein E (2013) Normal muscle regeneration requires tight control of muscle cell fusion by tetraspanins CD9 and CD81. Nat Commun 4: 1674 doi:10.1038/ncomms2675

Charrin S, Le Naour F, Oualid M, Billard M, Faure G, Hanash SM, Boucheix C, Rubinstein E (2001) The major CD9 and CD81 molecular partner. Identification and characterization of the complexes. J Biol Chem 276: 14329-14337. doi:10.1074/jbc.M011297200

Charrin S, Le Naour F, Silvie O, Milhiet PE, Boucheix C, Rubinstein E (2009a) Lateral organization of membrane proteins: Tetraspanins spin their web. Biochem J 420: 133-154. doi:10.1042/BJ20082422

Charrin S, Manié S, Billard M, Ashman L, Gerlier D, Boucheix C, Rubinstein E (2003) Multiple levels of interactions within the tetraspanin web. Biochem Biophys Res Commun 304: 107-112. doi:10.1016/S0006291X(03)00545-X

Charrin S, Yalaoui S, Bartosch B, Cocquerel L, Franetich JF, Boucheix C, Mazier D, Rubinstein E, Silvie O (2009b) The Ig domain protein CD9P-1 downregulates CD81 ability to support Plasmodium yoelii infection. J Biol Chem 284: 31572-31578. doi:10.1074/jbc.M109.057927

Cunha ES, Sfriso P, Rojas AL, Hospital A, Orozco M, Abrescia NGA (2017) Mechanism of structural tuning of the hepatitis $C$ virus human cellular receptor CD81 large extracellular loop. Structure 25: 53-65. doi:10.1016/j.str.2016.11.003

Deneka M, Pelchen-Matthews A, Byland R, Ruiz-Mateos E, Marsh M (2007) In macrophages, HIV-1 assembles into an intracellular plasma membrane domain containing the tetraspanins CD81, CD9, and CD53. J Cell Biol 177: 329-341. doi:10.1083/jcb.200609050

Dornberger-Schiff K (1956) On order-disorder structures (OD-structures). Acta Crystallogr 9: 593-601. doi:10.1107/s0365110×56001625

Earnest JT, Hantak MP, Li K, McCray PB, Perlman S, Gallagher T (2017) The tetraspanin CD9 facilitates MERS-coronavirus entry by scaffolding host cell receptors and proteases. PLoS Pathog 13: 1-22. doi:10.1371/ journal.ppat.1006546
Emsley P, Cowtan K (2004) Coot: Model-building tools for molecular graphics Acta Crystallogr D Biol Crystallogr 60: 2126-2132. doi:10.1107/ S0907444904019158

Florin L, Lang T (2018) Tetraspanin assemblies in virus infection. Front Immunol 9: 1-9. doi:10.3389/fimmu.2018.01140

Goddard TD, Huang CC, Ferrin TE (2007) Visualizing density maps with UCSF Chimera. J Struct Biol 157: 281-287. doi:10.1016/j.jsb.2006.06.010

Gordón-Alonso M, Yañez-Mó M, Barreiro O, Álvarez S, Muñoz-Fernández MÁ, Valenzuela-Fernández A, Sánchez-Madrid F (2006) Tetraspanins CD9 and CD81 modulate HIV-1-Induced membrane fusion. J Immunol 177: 5129-5137. doi:10.4049/jimmunol.177.8.5129

Grove J, Hu K, Farquhar MJ, Goodall M, Walker L, Jamshad M, Drummer HE, Bill RM, Balfe P, McKeating JA (2017) A new panel of epitope mapped monoclonal antibodies recognising the prototypical tetraspanin CD81. Wellcome Open Res 2: 1-17. doi:10.12688/ wellcomeopenres.12058.1

Gutiérrez-López MD, Gilsanz A, Yàñez-Mó M, Ovalle S, Lafuente EM, Dominguez C, Monk PN, Gonzàlez-Alvaro I, Sànchez-Madrid F, Cabañas C (2011) The sheddase activity of ADAM17/TACE is regulated by the tetraspanin CD9. Cell Mol Life Sci 68: 3275-3292. doi:10.1007/s00018011-0639-0

Hemler ME (2005) Tetraspanin functions and associated microdomains. Nat Rev Mol Cell Biol 6: 801-811. doi:10.1038/nrm1736

Homsi Y, Lang T (2017) The specificity of homomeric clustering of CD81 is mediated by its $\delta$-loop. FEBS Open Bio 7: 274-283. doi:10.1002/22115463.12187

Homsi Y, Schloetel JG, Scheffer KD, Schmidt TH, Destainville N, Florin L, Lang T (2014) The extracellular $\delta$-domain is essential for the formation of CD81 tetraspanin webs. Biophys / 107: 100-113. doi:10.1016/ j.bpj.2014.05.028

Ishii M, Iwai K, Koike M, Ohshima S, Kudo-Tanaka E, Ishii T, Mima T, Katada Y, Miyatake K, Uchiyama Y, et al (2006) RANKL-induced expression of tetraspanin CD9 in lipid raft membrane microdomain is essential for cell fusion during osteoclastogenesis. J Bone Miner Res 21: 965-976. doi:10.1359/jbmr.060308

Iwamoto R, Higashiyama S, Mitamura T, Taniguchi N, Klagsbrun M, Mekada E (1994) Heparin-binding EGF-like growth factor, which acts as the diphtheria toxin receptor, forms a complex with membrane protein DRAP27/CD9, which up-regulates functional receptors and diphtheria toxin sensitivity. EMBO J 13: 2322-2330. doi:10.1002/j.14602075.1994.tb06516.x

Kaji K, Oda S, Shikano T, Ohnuki T, Uematsu Y, Sakagami J, Tada N, Miyazaki S, Kudo A (2000) The gamete fusion process is defective in eggs of Cd9deficient mice. Nat Genet 24: 279-282. doi:10.1038/73502

Kitadokoro K, Bordo D, Galli G, Petracca R, Falugi F, Abrignani S, Grandi G, Bolognesi M (2001) CD81 extracellular domain 3D structure: Insight into the tetraspanin superfamily structural motifs. EMBO J 20: 12-18. doi:10.1093/emboj/20.1.12

Kitadokoro K, Ponassi M, Galli G, Petracca R, Falugi F, Grandi G, Bolognesi M (2002) Subunit association and conformational flexibility in the head subdomain of human CD81 large extracellular loop. Biol Chem 383: 1447-1452. doi:10.1515/BC.2002.164

Kovalenko OV, Metcalf DG, DeGrado WF, Hemler ME (2005) Structural organization and interactions of transmembrane domains in tetraspanin proteins. BMC Struct Biol 5: 1-20. doi:10.1186/1472-6807-511

Liu Y, Eisenberg D (2002) 3D domain swapping: As domains continue to swap. Protein Sci 11: 1285-1299. doi:10.1110/ps.0201402

Lutz M, Kroon-Batenburg LMJ (2018) Order-disorder in diaquobis(salicylato) copper(II) revisited. Croat Chem Acta 91: 289-298. doi:10.5562/cca3362

Machado-Pineda Y, Cardeñes B, Reyes R, López-Martín S, Toribio V, SánchezOrganero P, Suarez H, Grötzinger J, Lorenzen I, Yáñez-Mó M, et al (2018) 
CD9 controls integrin $\alpha 5 \beta 1$-mediated cell adhesion by modulating its association with the metalloproteinase ADAM17. Front Immunol 9: 1-14. doi:10.3389/fimmu.2018.02474

Maecker T, Todd C, Levy S (1997) The tetraspanin superfamiliy: Molecular facilitators. FASEB J 11: 428-442. doi:10.1096/fasebj.11.6.9194523

McCoy AJ, Grosse-Kunstleve RW, Adams PD, Winn MD, Storoni LC, Read RJ (2007) Phaser crystallographic software. J Appl Crystallogr 40: 658-674. doi:10.1107/S0021889807021206

Murshudov GN, Skubák P, Lebedev AA, Pannu NS, Steiner RA, Nicholls RA, Winn MD, Long F, Vagin AA (2011) REFMAC5 for the refinement of macromolecular crystal structures. Acta Crystallogr D Biol Crystallogr 67: 355-367. doi:10.1107/S0907444911001314

Nakamura K, Iwamoto R, Mekada E (1995) Membrane-anchored heparinbinding EGF-like growth factor (HB-EGF) and diphtheria toxin receptor-associated protein (DRAP27)/CD9 form a complex with integrin $\alpha 3 \beta 1$ at cell-cell contact sites. J Cell Biol 129: 1691-1705. doi:10.1083/jcb.129.6.1691

Nelson B, Adams J, Kuglstatter A, Li Z, Harris SF, Liu Y, Bohini S, Ma H, Klumpp K, Gao J, et al (2018) Structure-guided combinatorial engineering facilitates affinity and specificity optimization of anti-CD81 antibodies. J Mol Biol 430: 2139-2152. doi:10.1016/j.jmb.2018.05.018

Neviani V (2019) Unravelling the molecular mechanisms of tetraspanin CD9. In Thesis, Crystal and Structural Chemistry, Bijvoet Centre for Biomolecular Research, Utrecht University, Utrecht, The Netherlands. pp 63-106.

Neviani V, van Deventer S, Wörner TP, Xenaki KT, van de Waterbeemd M, Rodenburg RNP, Wortel IMN, Kuiper JK, Huisman S, Granneman J, et al (2020) Site-specific functionality of lipidation in tetraspanin CD9 revealed by tryptophan mimicry. FEBS J 63-106. doi:10.1111/febs.15295

Odintsova E, Voortman J, Gilbert E, Berditchevski F (2003) Tetraspanin CD82 regulates compartmentalisation and ligand-induced dimerization of EGFR. J Cell Sci 116: 4557-4566. doi:10.1242/jcs.00793

Potterton L, Agirre J, Ballard C, Cowtan K, Dodson E, Evans PR, Jenkins HT, Keegan R, Krissinel E, Stevenson K, et al (2018) CCP4i2: The new graphical user interface to the CCP4 program suite. Acta Crystallogr D Struct Biol 74: 68-84. doi:10.1107/S2059798317016035

Reyes R, Cardeñes B, Machado-Pineda Y, Cabañas C (2018) Tetraspanin CD9: A key regulator of cell adhesion in the immune system. Front Immunol 9: 1-9. doi:10.3389/fimmu.2018.00863

Reyes R, Monjas A, Yánez-Mó M, Cardeñes B, Morlino G, Gilsanz A, MachadoPineda Y, Lafuente E, Monk P, Sánchez-Madrid F, et al (2015) Different states of integrin LFA-1 aggregation are controlled through its association with tetraspanin CD9. Biochim Biophys Acta 1853: 2464-2480. doi:10.1016/j.bbamcr.2015.05.018

Rosenthal PB, Henderson R (2003) Optimal determination of particle orientation, absolute hand, and contrast loss in single-particle electron cryomicroscopy. J Mol Biol 333: 721-745. doi:10.1016/ j.jmb.2003.07.013

Rubinstein E, le Naour F, Billard M, Prenant M, Boucheix C (1994) CD9 antigen is an accessory subunit of the VLA integrin complexes. Eur I Immunol 24: 3005-3013. doi:10.1002/eji.1830241213

Rubinstein E, Le Naour F, Lagaudrière-Gesbert C, Billard M, Conjeaud $\mathrm{H}_{\text {, }}$ Boucheix C (1996) CD9, CD63, CD81, and CD82 are components of a surface tetraspan network connected to HLA-DR and VLA integrins. Eur J Immunol 26: 2657-2665. doi:10.1002/eji.1830261117

Sala-Valdés M, Ursa Á, Charrin S, Rubinstein E, Hemler ME, Sánchez-Madrid F, Yáñez-Mó M (2006) EWI-2 and EWI-F link the tetraspanin web to the actin cytoskeleton through their direct association with ezrin-radixinmoesin proteins. J Biol Chem 281: 19665-19675. doi:10.1074/ jbc.M602116200

Scheres SHW (2015) Semi-automated selection of cryo-EM particles in RELION-1.3. J Struct Biol 189: 114-122. doi:10.1016/j.jsb.2014.11.010
Scheres SHW (2012) RELION: Implementation of a Bayesian approach to cryoEM structure determination. J Struct Biol 180: 519-530. doi:10.1016/ j.jsb.2012.09.006

Schmidt TH, Homsi Y, Lang T (2016) Oligomerization of the tetraspanin CD81 via the flexibility of its $\delta$-loop. Biophys / 110: 2463-2474. doi:10.1016/ j.bpj.2016.05.003

Schreurs AMM, Xian X, Kroon-Batenburg LMJ (2010) EVAL15: A diffraction data integration method based on ab initio predicted profiles. J Appl Crystallogr 43: 70-82. doi:10.1107/S0021889809043234

Seigneuret M, Delaguillaumie A, Lagaudrière-Gesbert C, Conjeaud H (2001) Structure of the tetraspanin main extracellular domain: A partially conserved fold with a structurally variable domain insertion. J Biol Chem 276: 40055-40064. doi:10.1074/jbc.M105557200

Sheldrick G (2009) Twinabs. University of Göttingen: Germany.

Stipp CS, Kolesnikova TV, Hemler ME (2001a) EWI-2 is a major CD9 and CD81 partner and member of a novel Ig protein subfamily. I Biol Chem 276: 40545-40554. doi:10.1074/jbc.M107338200

Stipp CS, Orlicky D, Hemler ME (2001b) FPRP, a major, highly stoichiometric, highly specific CD81- and CD9-associated protein. J Biol Chem 276: 4853-4862. doi:10.1074/jbc.M009859200

Tachibana I, Hemler ME (1999) Role of transmembrane 4 superfamily (TM4SF) proteins CD9 and CD81 in muscle cell fusion and myotube maintenance. J Cell Biol 146: 893-904. doi:10.1083/jcb.146.4.893

Takeda Y, Tachibana I, Miyado K, Kobayashi M, Miyazaki T, Funakoshi T, Kimura H, Yamane H, Saito Y, Goto H, et al (2003) Tetraspanins CD9 and CD81 function to prevent the fusion of mononuclear phagocytes. I Cell Biol 161: $945-956$. doi:10.1083/jcb.200212031

Tang G, Peng L, Baldwin PR, Mann DS, Jiang W, Rees I, Ludtke SJ (2007) EMAN2: An extensible image processing suite for electron microscopy. J Struct Biol 157: 38-46. doi:10.1016/j.jsb.2006.05.009

Tickle I, Flensburg C, Keller P, Paciorek W, Sharff A, Smart O, Vonrhein C, Bricogne G (2018) STARANISO. Cambridge, UK: Global Phasing Ltd.

Umeda R, Nishizawa T, Nureki O (2019) Crystallization of the human tetraspanin protein CD9. Acta Crystallogr F Struct Biol Commun 75: 254-259. doi:10.1107/S2053230X1801840X

Umeda R, Satouh Y, Takemoto M, Nakada-nakura Y, Liu K, Yokoyama T, Shirouzu M, Iwata S, Nomura N, Sato K, et al (2020) Structural insights into tetraspanin CD9 function. Nat Commun 11: 1606. doi:10.1038/ s41467-020-15459-7

Van Deventer SJ, Dunlock VME, Van Spriel AB (2017) Molecular interactions shaping the tetraspanin web. Biochem Soc Trans 45: 741-750. doi:10.1042/BST20160284

Waterhouse A, Bertoni M, Bienert S, Studer G, Tauriello G, Gumienny R, Heer FT, De Beer TAP, Rempfer C, Bordoli L, et al (2018) SWISS-MODEL: Homology modelling of protein structures and complexes. Nucleic Acids Res 46: W296-W303. doi:10.1093/nar/gky427

Winter G, Waterman DG, Parkhurst JM, Brewster AS, Gildea RJ, Gerstel M, Fuentes-Montero L, Vollmar M, Michels-Clark T, Young ID, et al (2018) DIALS: Implementation and evaluation of a new integration package. Acta Crystallogr D Struct Biol 74: 85-97. doi:10.1107/S2059798317017235

Yáñez-Mó M, Barreiro O, Gordon-Alonso M, Sala-Valdés M, Sánchez-Madrid F (2009) Tetraspanin-enriched microdomains: A functional unit in cell plasma membranes. Trends Cell Biol 19: 434-446. doi:10.1016/j.tcb.2009.06.004

Yang W, Zhang M, Chi X, Liu X, Qin B, Cui S (2015) An intramolecular bond at cluster of differentiation 81 ectodomain is important for hepatitis $C$ virus entry. FASEB J 29: 4214-4226. doi:10.1096/fj.15-272880

Yang Y, Liu XR, Liu S, Shen G, Li W, Greenberg ZJ, Zhou F, He P, Fan L, Egawa T, et al (2020) Open conformation of tetraspanins shapes interaction partner networks on cell membranes. EMBO J e105246. doi:10.15252/embj.2020105246

Zhang K (2016) Gctf: Real-time CTF determination and correction. J Struct Biol 193: 1-12. doi:10.1016/j.jsb.2015.11.003 
Zheng SQ, Palovcak E, Armache J-P, Verba KA, Cheng Y, Agard DA (2017) MotionCor2: Anisotropic correction of beam-induced motion for improved cryo-electron microscopy. Nat Methods 14: 332-333. doi:10.1038/nmeth.4193

Zimmerman B, Kelly B, McMillan BJ, Seegar TCM, Dror RO, Kruse AC, Blacklow SC (2016) Crystal structure of a full-length human tetraspanin reveals a cholesterolbinding pocket. Cell 167: 1041-1051.e11. doi:10.1016/j.cell.2016.09.056

Zuidscherwoude M, de Winde CM, Cambi A, van Spriel AB (2014)

Microdomains in the membrane landscape shape antigen-presenting cell function. J Leukoc Biol 95: 251-263. doi:10.1189/jlb.0813440
Zuidscherwoude M, Göttfert F, Dunlock VME, Figdor CG, Van Den Bogaart G, Van Spriel AB (2015) The tetraspanin web revisited by super-resolution microscopy. Sci Rep 5: 1-18. doi:10.1038/ srep12201

(c)

License: This article is available under a Creative Commons License (Attribution 4.0 International, as described at https://creativecommons.org/ licenses/by/4.0/). 